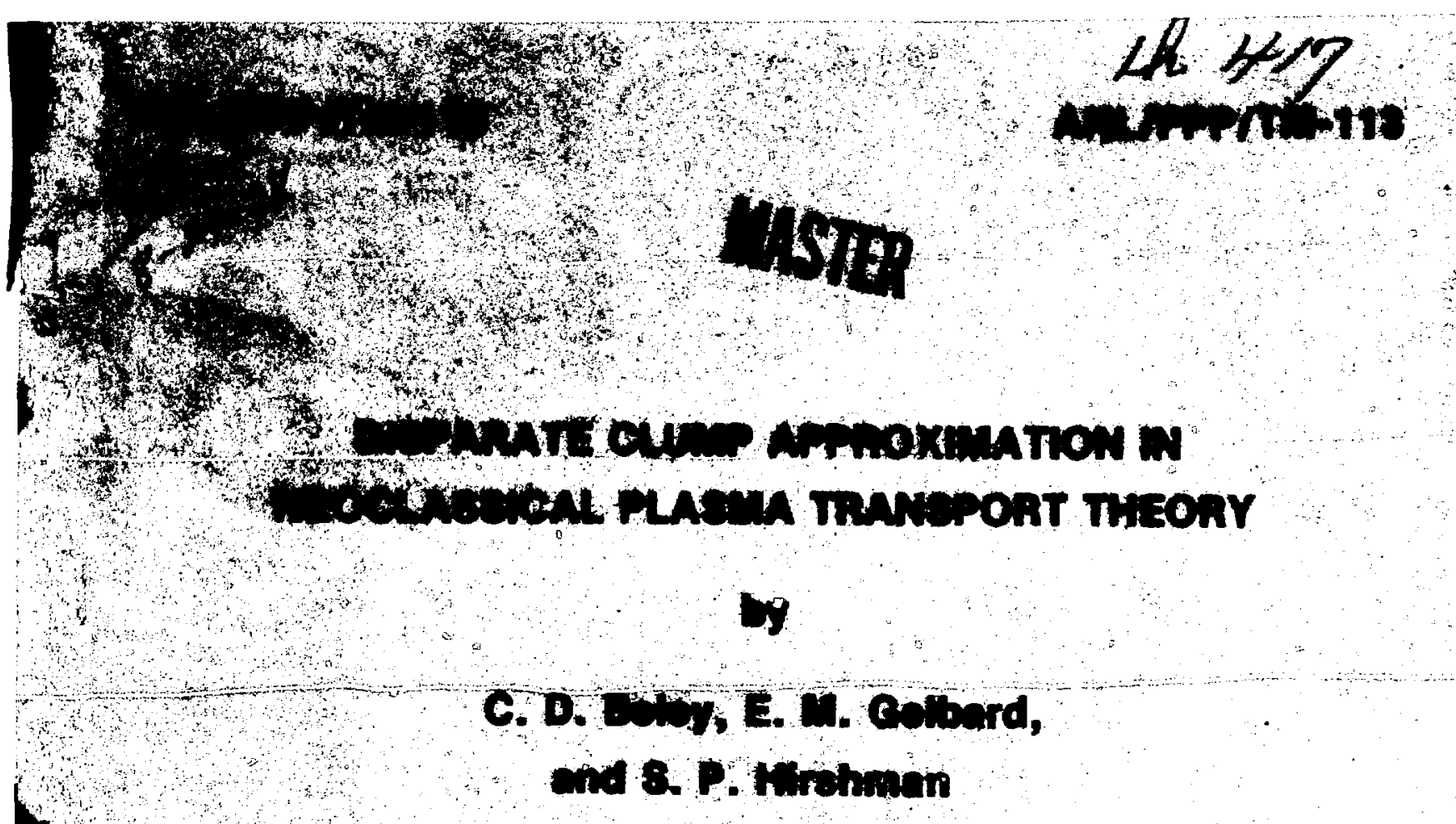


ANL/FPP/TM-113

\author{
ARGONNE NATIONAL LABORATORY \\ 9700 South Cass Avenue \\ Argonne, Illinois 60439
}

\title{
DISPARATE CLUMP APPROXIMATION IN NEOCLASSICAL PLASMA TRANSPORT THEORY
}

by

C. D. Boley and E. M. Gelbard

Applied Physics Division

Argonne National Laboratory

and

S. P. Hirshman*

Plasma Physios Laboratory

Princeton University

Princeton, New Jersey

July 1978

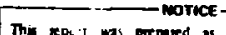

The rept T wat prepared as en eccount of work

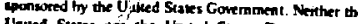

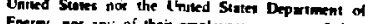
Energ, nor any of their employers, nor s'y of the conitsctons. aubcontractors, ot thetr entiptoy ses. makes

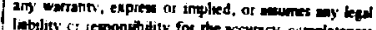

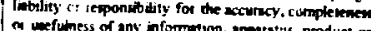
at unefuness of any informition. appatat 13 . product or

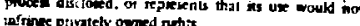

Results reported in the FPP series of memoranda frequently are preliminary and subject to revision.

*Present address: Oak Ridge National Laboratory, Oak Ridge, Tennessee 37830 


\section{TABLE OF CONTENTS}

Page

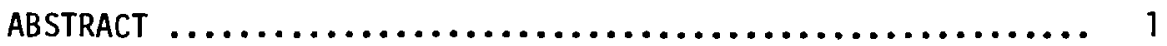

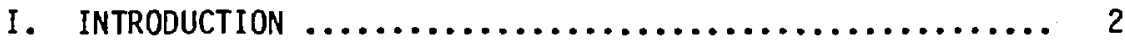

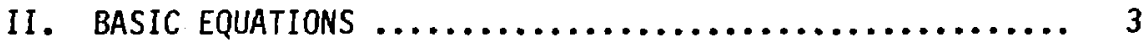

II . COULOMB MATRIX ELEMENTS IN A DISPARATE CLUMP SYSTEM $\ldots . .6$

IV. SOLUTION OF THE SPITZER PROBLEM - FIRST FORMULATION $\ldots 12$

v. SOLUTION OF THE SPITZER PROBLEM - SECOND FORMULATION .. 19

A. Single-Clump System $\ldots \ldots \ldots \ldots \ldots \ldots \ldots \ldots \ldots \ldots \ldots . . . \ldots$

B. Properties of $\Delta, \alpha$, and $\beta$ for Disparate-Mass Species 24

C. Computation of $\ell_{11}, \ell_{12}, \ell_{22}$, and $H \ldots \ldots \ldots \ldots \ldots, 26$

D. Computation of the Force on a Species: Parallel Velocities ................................ 28

E. Random Heat Fluxes ......................... 31

VI. DIFFUSION COEFFICIENTS IN THE PFIRSCH-SCHL̈̈TER REgIME .. 32

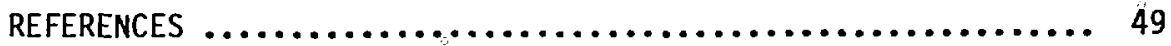




\title{
DISPARATE CLUMP APPROXIMATION IN \\ NEOCLASSICAL PLASMA TRANSPORT THEORY
}

by

C. D. Boley, E. M. Gelbard, and S. P. Hirshman

\begin{abstract}
We consider a model of a multiple ion plasma in which the system may contain many atomic species, each with an arbitrary number of charge states, but in which the atomic species are disparate in mass. Using this approximation we calculate, in analytic form, the multispecies Spitzer function, from which there follow tractable expressions for the Ware fluxes and bootstrap current in a tokamak. In the collisional regime of a tokamak plasma, we obtain simple analytic results for the radial transport coefficients. These results give rise to an efficient method of solving the ensuing diffusion equations.
\end{abstract}




\section{INTRODUCTION}

Typical tokamak discharges show evidence of various impurity ions, often with a number of charge states. In TFR, for example, radial profiles have been measured for several charge states of oxygen, ${ }^{(1)}$ while in PLT much attention has been focused on tungsten impurities. ${ }^{(2)}$ In plasma transport calculations for such systems, it is sometımes necessary to account for the transport of different charge states separately, without invoking the coronal approximation. The appropriate neoclassical transport coefficients have been worked out in a variety of collisionality regimes. $(3-10)$ Standing in the way of their implementation into one-dimensional codes are two problems. First, the computation of the transport coefficients themselves consumes a great deal of time. In the Pfirsch-Schlüter regime, for example, this requires the inversion of matrices with" dimensions of $(N+2) \times(N+2)$, where $N$ is the total number of ion species (including charge states) and where it is assumed that all ions have the same temperature. Secord, even if values of the transport coefficients are given, running times still increase rapidly with $N$ since $N+2$ diffusion equations must be solved simultaneously.

In this report we describe a "disparate clump" approximation which is expected to alleviate these problems. Briefly, we consider a multiple ion system in which each ion can have an arbitrary number of charge states, but in which the ion masses are assumed to be disparate; all the charge states of a given ion, of course, have the same mass. By "disparate" masses we mean that the masses can be ordered such that $m_{1} \ll m_{2} \ll m_{3}$. . . We refer collectively to the charge states of an ion as a clump. Clearly the electrons can be taken as the first clump.

We develop the disparate clump approximation in two specific contexts. First, we employ it in caiculating the classical Spitzer function (response of 
the distribution function to a parallel electric field) for a multiple ion system. Our motivation here is the fact that the neoclassical transport coefficients giving the Ware pinch and bootstrap current require the Spitzer function as input. $(5,10)$ With the aid of the results obtained here, these transport coefficients can readily be ccmputed.

In the second context, we consider a multiple ion plasma in the PfirschSchlüter regime, where the transport coefficients can be expressed in terms of the response to parallel pressure and temperature gradients. ${ }^{(6)}$ Using the disparate clump approximation, we obtain simple analytic results for the tranport coefficients. Furthermore, certain special features of these expressions will be shown to suggest an efficient method of solving the associated one-dimensional diffusion equations, thus alleviating the problem of long running times.

The organization of this paper is as follows. In the next section we set up the basic equations which describe the problems under consideration. In Section III we give the special form of the Coulomb collision "operator appropriate to a disparote clump system. Using the collision operator in this form, we solve for the Spitzer function in Section IV. Anuther derivation, from a somewhat different point of view, is presented in Section V. Finally, in Section VI we work out the diffusion problem in the Pfirsch-Schlüter regime.

\section{BASIC EQUATIONS}

The problems to be considered in this report are described by the basic equation

$$
v_{1} I_{\alpha} f_{\alpha 0}=\sum_{\beta}\left(c_{\alpha \beta}\left[f_{\alpha 1}, f_{\beta 0}\right]+C_{\alpha \beta}\left[f_{\alpha 0}, f_{\beta 1}\right]\right),
$$

where the indices $\alpha$ and $\beta$ label the plasma species, $C_{\alpha \beta}$ is the Coulomb collision operator, $v_{1}$ is the component of the velocity $\vec{v}$ parallel to the local 
magnetic field, and $f_{\alpha 0}$ is a local Maxwellian distribution function without drift:

$$
f_{\alpha 0}(v)=n_{\alpha}\left(\pi v_{T \alpha}^{2}\right)^{-3 / 2} e^{-v^{2} / v_{T \alpha}^{2}}, \quad v_{T \alpha}^{2}=\frac{2 T_{\alpha}}{m_{\alpha}} .
$$

The distribution function has been written as $f_{\alpha}=f_{\alpha 0}+f_{\alpha l}$, and the collision operator correspondingly linearized. The quantity $I_{\alpha}$ is a driving term whose definition depends on the problem under investigation. In the Spitzer problem we have

$$
I_{\alpha}^{(S)}=-\frac{e_{\alpha} E_{1}}{T_{\alpha}}
$$

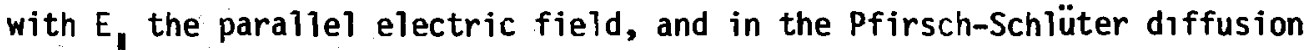
problem we have

$$
I_{\alpha}^{(P S)}=\nabla_{1} \text { ln } p_{\alpha}-\left(\frac{5}{2}-x_{\alpha}^{2}\right) \nabla_{1} \text { en } T_{\alpha},
$$

with $x_{\alpha}^{2}=\left(v / v_{T \alpha}\right)^{2}, p_{\alpha}=n_{\alpha}^{\top}{ }_{\alpha}$, and $\nabla_{1}$ the parallel gradient.

If the operation $\sum_{\alpha} m_{\alpha} \int d^{3} v v_{1}$ is applied to Eq. (2.1), the right-hand side vanishes by virtue of momentum conservation and we are left with the solvability conditions for the two problems. In the Spitzer problem this is the condition of local charge neutrality,

$$
\sum_{\alpha} n_{\alpha} e_{\alpha}=0
$$

and in the diffusion problem it is the condition that the total pressure be constant along a magnetic field line,

$$
\nabla_{1} \sum_{\alpha} p_{\alpha}=0
$$


The latter is, of course, in accord with the equilibrium MHD equation $\vec{\nabla} p=\vec{J} \times \dot{B}$.

To solve the kinetic equation, we approximate the perturbed distribution function as a truncated expansion in energy-space polynomials:

$$
f_{\alpha 1}=\frac{2 v_{1}}{v_{T \alpha}^{2}} f_{\alpha 0} \sum_{k=0}^{k} u_{\alpha}^{(k)} L_{k}^{(3 / 2)}\left(x_{\alpha}^{2}\right)
$$

with $L_{k}^{(3 / 2)}$ an associated Laguerre polynomial. The first moment $u_{\alpha}^{(0)}$ is the parallel flow velocity, and the second is $u^{(1)}=-\left(2 q_{1 \alpha} / 5 n_{\alpha} T_{\alpha}\right)$, with $q_{1 \alpha}$ the random heat flux through species $\alpha$. The solution is determined by the first $k_{\max }+1$ mements of Eq. (2.1) with respect to $v_{1} L_{k}^{(3 / 2)}\left(x_{\alpha}^{2}\right)$. In the Spitzer problem the resulting system is

$$
\sum_{\beta} \sum_{k^{\prime}=0}^{k} Q_{\alpha \beta}^{k k^{\prime}} u_{\beta}^{\left(k^{\prime}\right)}=-\delta_{k 0} n_{\alpha} e_{\alpha} E_{1} \text {, }
$$

and in the diffusion problem it is

$$
\sum_{\beta} \sum_{k^{\prime}=0}^{k} Q_{\alpha \beta}^{k k^{\prime}} u_{\beta}^{\left(k^{\prime}\right)}=\delta_{k 0} \nabla_{1} p_{\alpha}-\frac{5}{2} \delta_{k 1} n_{\alpha} \nabla_{1} T_{\alpha} \text {, }
$$

where $Q_{\alpha \beta}^{k^{\prime}}$ is defined in terms of the matrix elements of the Coulomb collision operator as follows:

$$
Q_{\alpha \beta}^{k k^{\prime}}=\delta_{\alpha \beta^{\prime} m_{\alpha}} \sum_{\gamma} M_{\alpha \gamma}^{k^{\prime}}+m_{\alpha} \frac{v_{T \alpha}}{v_{T \beta}} \mu_{\alpha \beta}^{k^{\prime}},
$$

with

$$
m_{\alpha \beta}^{k k}=\int d^{3} v \frac{v_{1}}{v_{T \alpha}} L_{k}^{(3 / 2)}\left(x_{\alpha}^{2}\right) C_{\alpha \beta}\left[\frac{2 v_{t}}{v_{T \alpha}} L_{k}^{(3 / 2)}\left(x_{\alpha}^{2}\right) f_{\alpha 0}, f_{\beta 0}\right],
$$




$$
N_{\alpha \beta}^{k k}=\int d^{3} v \frac{v_{1}}{v_{T \alpha}} L_{k}^{(3 / 2)}\left(x_{\alpha}^{2}\right) C_{\alpha \beta}\left[f_{\alpha 0}, \frac{2 v_{1}}{v_{T \beta}} L_{k^{-}}^{(3 / 2)}\left(x_{\beta}^{2}\right) f_{\beta 0}\right] .
$$

In terms of $Q_{\alpha \beta}^{k^{-}}$, the statement of momentum conservation is

$$
\sum_{\alpha} Q_{\alpha \beta}^{0 k^{-}}=0
$$

We proceed to a discussion of $Q_{\alpha \beta}^{k^{-}}$in a disparate clump system.

\section{COULOMB MATRIX ELEMENTS IN A DISPARATE CLUMP SYSTEM}

We label the clumps by $a, b$, etc. and the charge states within a clump by $i, j$. Thus the density and charge of charge state $\underline{i}$ within clump a are denoted $n_{a 1}$ and $e_{a 1}$. Al1 charge states within the clump have the same mass $m_{a}$ and temperature $T_{a}$. The clumps are assumed to be ordered such that $m_{1} \ll m_{2} \ll$ ... $m_{\mathrm{N}}$, the first "clump" being the electrons.

We turn first to the matrix elements of M. Taking $m_{a} \ll m_{b}$, we have

$$
M_{a i, b j}^{k k^{\prime}}=-n_{a i} \nu_{a i, b j}{ }^{k k^{\prime}}, \quad\left(m_{a} \ll " m_{b}\right),
$$

where $\nu_{a i, b j}$ is the Braginskii collision frequency,

$$
v_{a i, b j}=\frac{4(2 \pi)^{1 / 2} e_{a i}^{2} e_{b j}^{2} n_{b j} \text { en } \Lambda}{3\left(4 \pi \varepsilon_{0}\right)^{2} m_{a}^{1 / 2} T_{a}^{3 / 2}} \text {, }
$$

and 


$$
\mu_{1}^{k k^{-}}=\int_{0}^{\infty} d t e^{-t} L_{k}^{(3 / 2)}(t) L_{k^{-}}^{(3 / 2)}
$$

$$
=\left(\begin{array}{cccc}
1 & \frac{3}{2} & \frac{15}{8} \\
\frac{3}{2} & \frac{13}{4} & \frac{69}{16} \\
\frac{15}{8} & \frac{69}{16} & \frac{433}{64} & .
\end{array}\right) \text {. }
$$

In the opposite limit, $m_{a} \gg m_{b}$, this matrix element becomes

$$
M_{a i, b j}^{k k}=-\frac{m_{b}}{m_{a}} n_{b j} v_{b j, a i}\left[\mu_{3}^{k k^{-}}+\left(\frac{T_{b}}{T_{a}}-1\right) \mu_{4}^{k k^{-}}\right], \quad\left(m_{a}>m_{b}\right)
$$

where

$$
\begin{aligned}
\mu_{3}^{k k^{\prime}} & =\frac{4 \Gamma(k+5 / 2)}{3 \sqrt{\pi} k !} \delta_{k^{\prime}}+\frac{8}{3 \sqrt{\pi}} \int_{0}^{\infty} d t e^{-t_{t}} \frac{5 / 2}{d L_{k}^{(3 / 2)}(t)} \frac{d L_{k^{\prime}}^{(3 / 2)}(t)}{d t} \\
& =\left(\begin{array}{ccc}
1 & 0 & 0 \\
0 & \frac{15}{2} & 0 \\
0 & 0 & \frac{175}{8}
\end{array}\right),
\end{aligned}
$$

and 


$$
\mu_{4}^{k k^{-}}=\mu_{3}^{k k^{-}}-\frac{4 \Gamma(k+5 / 2)}{3 \sqrt{\pi} k !}\left[(2 k+1) \delta_{k k^{-}}-2 k \delta_{k-1, k^{-}}\right]
$$

$$
=\left(\begin{array}{cccccc}
0 & 0 & 0 & & \\
5 & 0 & 0 & & \\
0 & \frac{35}{2} & 0 & & \\
& & & & .
\end{array}\right) \cdot
$$

The matrix element of $M$ given in Eq. (3.4) is of higher order in the mass ratio than the matrix elements in Eq. (3.1), and it will not contribute to the calculations at hand. Finally, the matrix element between charge states of the same ion has the form

$$
M_{a 1, a j}^{k k^{\prime}}=n_{a 1}{ }_{a 1, a j}{ }^{m^{k k^{\prime}}},
$$

where

$$
\begin{aligned}
m^{k k^{-}}= & -\int \frac{d^{3} x d^{3} x^{-}}{4 \pi^{5 / 2}} e^{-x^{2}-x^{-2} T_{1 \ell}^{k}(\vec{x}) U_{i j}\left(\vec{x}-\vec{x}^{-}\right) T_{j l^{k}}^{k^{-}}(\vec{x}-)} \\
= & -\frac{1}{1}\left(\begin{array}{ccc}
1-\frac{3}{4} & \frac{15}{32} \\
\frac{3}{4} & \frac{59}{16} & \frac{417}{128} \\
\frac{15}{32} & \frac{417}{128} & \frac{8385}{1024}
\end{array}\right) .
\end{aligned}
$$

We have used the notation 


$$
\begin{aligned}
& T_{1 \ell}^{k}(\vec{x})=\frac{\partial}{\partial x_{1}}\left[x_{\ell} L_{k}^{(3 / 2)}\left(x^{2}\right)\right], \\
& U_{1 j}(\vec{x})=\frac{\delta_{1 j}^{x^{2}-x_{1} x_{j}}}{x^{3}} .
\end{aligned}
$$

Evaluating the matrix elements of the other part/ of the collision operator, we have

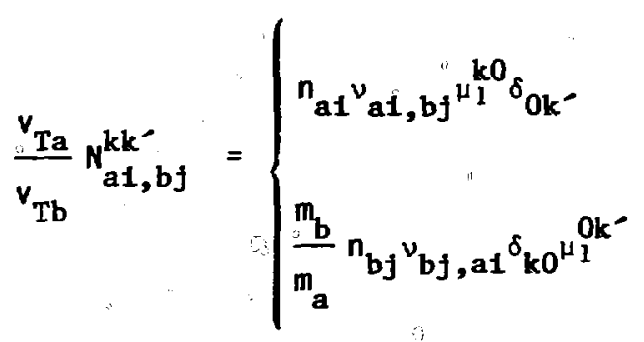

$$
m_{a}>m_{b}
$$

and, within a clump,

$$
N_{a 1, b j}^{k k^{-}}=n_{a 1}^{\nu}{ }_{a 1, b j} n^{k k^{-}},
$$

where

$$
n^{k k^{-}}=-\sqrt{2} \mu_{2}^{k k^{-}}-m^{k k^{-}},
$$

with

9 


$$
\begin{aligned}
\mu_{2}^{k k^{-}} & =\left(\frac{d^{3} x d^{3} x^{-}}{2(2 \pi)^{5 / 2}} e^{-x^{2}-x^{-2}}\left[T_{i \ell}^{k}(\vec{x})-T_{i \ell}^{k}\left(\vec{x}^{-}\right)\right] U_{i j}\left(\vec{x}-\vec{x}^{-}\right)\left[T_{j \ell}^{k^{-}}(\vec{x})-T_{j \ell^{k}}^{k^{-}}\left(\vec{x}^{-}\right)\right]\right. \\
& =\left(\begin{array}{ccc}
0 & 0 & 0 \\
0 & 1 & \frac{3}{4} \\
0 & \frac{3}{4} & \frac{45}{16}
\end{array}\right)
\end{aligned}
$$

Putting all this information together, we obtain the following expression for $Q_{a i, b j}^{k k}$ :

$$
\begin{aligned}
& Q_{a i, b j}^{k k^{-}}=-\delta_{a b} \delta_{i j}\left\{\lambda_{a i, a} D^{k k^{-}}\left(Z_{a}\right)+\sum_{c<a} \lambda_{c \ell, a i}\left[\mu_{3}^{k k^{-}}+\left(\frac{T_{c}}{T_{a}}-1\right) \mu_{4}^{k k^{-}}\right]\right\} \\
& +\delta_{a b} \lambda_{a i, a j} n^{k k^{-}} \\
& +\theta_{a b^{\lambda}}{ }_{a i, b j} \mu_{1}^{k 0} \delta_{0 k^{-}}+\theta_{b a} \lambda_{b j, a i} \delta_{k 0^{\mu_{1}}} k^{-},
\end{aligned}
$$

where

$$
\begin{aligned}
\lambda_{a i, b j} & =m_{a} n_{a i}{ }^{\nu} a i, b j \\
\lambda_{a i, b} & =\sum_{j} \lambda_{a i, b j}, \\
z_{a} & =\left(\sum_{i} n_{a i} e_{a i}^{2}\right)^{-1} \sum_{b>a}^{j} n_{b j} e_{b j}^{2},
\end{aligned}
$$




$$
\theta_{a b}=\left\{\begin{array}{ll}
1, & a<b \\
0, & a \geq b
\end{array},\right.
$$

and finally

$$
D^{k^{k}}(Z)=-m^{k k^{-}}+Z_{\mu 1}^{k_{1}^{\prime \prime}} .
$$

It can be checked that this expression is consistent with the conservation of momen tum,

$$
\sum_{\mathbf{a i}} Q_{a i, b j}^{0 k^{-}}=0 \text {, }
$$

since $\mu_{2}^{0 \mathrm{k}^{-}}=\mu_{4}^{0 \mathrm{k}^{\prime}}=0$ and $\mu_{3}^{0 \mathrm{k}^{-}}=\delta_{0 \mathrm{k}^{-}}$.

It will be noted that $Q$, as given by Eq. (3.14), does not fulfill the symmetry condition $Q_{a i, b j}^{k k^{\prime}}=Q_{b j, a i}^{k \cdot k}$ because of the presence of the term

$$
\left(\frac{T_{c}}{T_{a}}-1\right)_{\mu_{4}}^{k^{-}}
$$

Henceforth we shall assume that the temperatures are sufficiently equilibrated for this term to be dropped. With a symmetric $Q$, then, we have the null condition

$$
\sum_{b j} Q_{a i, b j}^{k 0}=0 .
$$

Thus a term of the form $\delta_{k 0} w$, with $w$ arbitrary, can be added to the solutions of Eqs. (2.6a) and (2.6b). 


\section{SOLUTION OF THE SPITZER PROBLEM - FIRST FORMLATION}

Having obtained $Q_{a a^{k}, b_{j}}^{k^{\prime}}$ for the disparate-clump system, we are now equipped to solve the Spitzer problem, which in the clump notation takes the form

$$
\sum_{b j} \sum_{k^{\prime}=0}^{\max _{a i, b j}} Q_{b j}^{k k^{\prime}} u_{\left.u^{\prime}\right)}^{\left(k^{\prime}\right)}=-\delta_{k 0^{n} n_{a i} e_{a i} E_{1}} \text {. }
$$

We can write the solution as

$$
u_{\text {ai }}^{(k)}=s_{k 0} w+E_{k} \bar{u}_{a i}^{(k)}
$$

with $w$ an arbitrary constant. Remembering that we are working in the disparatemass limit, we see that the leading term of $u_{a i}^{(k)}$ is proportional to $\mathrm{m}^{-1 / 2}$, and we discard the corrections. To treat these corrections consistently, a more accurate expression for $Q$ would be needed. To the indicated order, we then have

$$
\begin{aligned}
& -\lambda_{a i, a} \sum_{k^{\prime}} D^{k k^{\prime}}\left(z_{a}\right)_{\bar{u}^{\left(k^{\prime}\right.}}+\sum_{j} \lambda_{a i, a j} \sum_{k^{\prime}} n^{k k^{\prime}} \bar{u}_{a j}^{\left(k^{\prime}\right)} \\
& +\delta_{k 0} \sum_{\substack{b<a \\
j}} \lambda_{b j, a i} \sum_{k^{\prime}} u_{l}^{k^{\prime}} \bar{u}_{b j}^{\left(k^{\prime}\right)}=-\delta_{k 0} n_{a i} e_{a i} E_{1},
\end{aligned}
$$

in which the sums over $k^{-}$run from zero to $k_{\max }$ *

Although this may look complicated, it simplifies after a summation over the charge-state index i. Doing the sum and bearing in mind the particular dependence of $\lambda_{a i, b j}$ on (ai) and $\left(b_{j}\right)$, we end up with an equation for the weighted unknown of a clump, defined by 


$$
U_{a}^{(k)}=\frac{1}{\zeta_{a}} \sum_{i} \zeta_{a i} \bar{u}_{a i}^{(k)}
$$

where

$$
\zeta_{a i}=n_{a i} e_{a i}^{2}, \quad \zeta_{a}=\sum_{i} \zeta_{a i} .
$$

This equation is

$$
-\lambda_{a a} \sum_{k^{\prime}} c^{k k^{\prime}}\left(z_{a}\right) u_{a}^{\left(k^{\prime}\right)}+\delta_{k 0} \sum_{b<a} \lambda_{b a} \sum_{k^{\prime}}{ }_{\mu_{1}}^{0 k^{\prime}} u_{b}^{\left(k^{\prime}\right)}=-\delta_{k 0} p_{a},
$$

with

$$
\begin{aligned}
c^{k^{\prime}}(z) & =\sqrt{2} \mu_{2}^{k^{\prime}}+z u_{1}^{k^{\prime}}, \\
\lambda_{a b} & =\sum_{i} \lambda_{a i, b j}, \\
\rho_{a} & =\sum_{i} n_{a i} e_{a i} .
\end{aligned}
$$

Letting $v_{a}^{(k)}=\lambda_{a a} U_{a}^{(k)}$, we can cast Eq. (4.5) into the simpler form

$$
\sum_{k^{\prime}} c_{a}^{k k^{-}} v_{a}^{\left(k^{\prime}\right)}=\delta_{k 0} \sum_{b<a}\left[\frac{\zeta_{a}}{\zeta_{b}} \sum_{k^{\prime}} \mu_{1}^{0 k^{\prime}} v_{b}^{\left(k^{\prime}\right)}+\rho_{a}\right] \text {, }
$$

with the abbreviation $\mathrm{C}_{\mathrm{a}}^{\mathbf{k k}^{-}}=\mathrm{C}^{\mathrm{kk} \mathrm{k}^{-}}\left(\mathrm{z}_{\mathrm{a}}\right)$.

He now specialize to the case in which clump a is different from the heaviest clump $(a<N)$. The case $a=N$ will be handled separately below. When $k \neq 0$, Eq. (4.7) becomes 


$$
\sum_{k^{\prime} \neq 0} c_{a}^{k k^{\prime}} v_{a}^{\left(k^{\prime}\right)}=-c_{a}^{k 0} v_{a}^{(0)}
$$

or

$$
v_{a}^{(k)}=-v_{a}^{(0)} \sum_{k \neq 0}\left(\bar{c}_{a}^{-1}\right)^{k k^{-}} c_{a}^{k^{-0} 0}
$$

where $\bar{C}_{a}^{k k^{-}}$means $C_{a}^{k k^{-}}$restricted to the space with $k$ and $k^{-}$different from zero. Now taking $k=0$ in Eq. (4.7) and using the above result, we find the recursion relation

$$
Z_{a} \alpha_{a} v_{a}^{(0)}=\sum_{b<a} \frac{\zeta_{a}}{\zeta_{b}} \alpha_{b} v_{b}^{(0)}+p_{a} \text {, }
$$

whers:

$$
\alpha_{a} \equiv \alpha\left(z_{a}\right)=1-z_{a} \sum_{\substack{k \neq 0 \\ k^{\circ} \neq 0}} \mu_{1}^{0 k}\left(\bar{c}_{a}^{-1}\right)^{k k^{-}} \mu_{1}^{k^{-0}} .
$$

This last quantity, which depends only on $z_{a}$ (and $k_{\max }$ ), is one of the Braginskii paral]el transport parameters. ${ }^{(12)}$ In the three-polynomial approximation $\left(k_{\max }=2\right)$, we have

$$
\begin{aligned}
& \alpha(z)=\left(1+\frac{61}{72} \sqrt{2} z+\frac{2}{9} z^{2}\right) / f(z), \\
& f(z)=1+\frac{151}{72} \sqrt{2} z+\frac{217}{288} z^{2} .
\end{aligned}
$$

To reduce the recursion relation, we let 


$$
r_{a}=\frac{z_{a} a_{a} v_{a}^{(0)}}{\rho_{a}}
$$

obtaining ${ }^{(5)}$

$$
r_{a}=1+\bar{e}_{a} \sum_{b<a}^{N} \frac{r_{b}}{\bar{e}_{b} z_{b}},
$$

with $\bar{e}_{a}=\zeta_{a} / \rho_{a}$. Converting this into a two-point difference equation, one can show that the solution is $(7,10)$

$$
r_{a}=1-\frac{1}{\rho_{a}\left(1+z_{a}\right)} \sum_{b=a}^{N} \rho_{b},
$$

in which charge neutrality has been used.

Turning to the case $a=N$, we note that Eq. (4.7) becomes, for $k \neq 0$,

$$
\sum_{k^{\prime} \neq 0} c_{N}^{k k^{-} v_{N}^{\left(k^{\prime}\right)}=0,}
$$

in view of the fact that $C_{\mathrm{N}}^{0 k}=C_{\mathrm{N}}^{\mathrm{k} 0}=0$. Since $C_{\mathrm{N}}$ is nonsingular on the restricted space, the solution is $v_{N}^{(k)}=0$. Next, when $k=0$, Eq. (4.7) becomes

$$
0=1+\frac{\zeta_{N}}{P_{N}} \sum_{b<N} \frac{r_{b}}{\bar{e}_{b} Z_{b}},
$$

which involves only the unknowns calculated in the previous paragraph, and which is satisfied identically. 'Thus $v_{\mathrm{N}}^{(0)}$ is undetermined, aside from the requirement that it be of order $\left(m_{N}\right)^{\frac{1}{2}}$

Summarizing the results thus far, we have: 


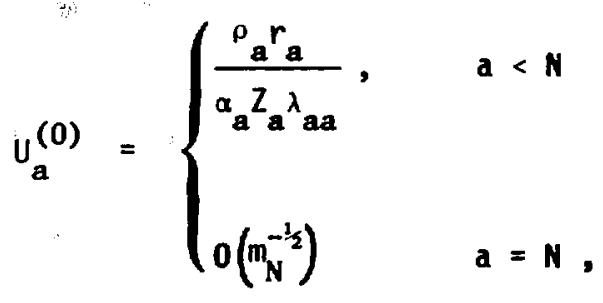

and for $k \neq 0$,

$$
u_{a}^{(k)}= \begin{cases}\left.U^{(0)}\right)_{R}^{(k)}\left(Z_{a}\right), & a<N \\ 0, & a=N,\end{cases}
$$

where

$$
R^{(k)}\left(z_{a}\right)=-z_{a} \sum_{k^{\prime} \neq 0}\left(\bar{c}_{a}^{-1}\right)^{k k^{-}} \mu_{1}^{k^{-0}} .
$$

In the three-polynomial approximation, we have

$$
\begin{aligned}
& R^{(1)}(Z)=-\frac{2}{5} \beta(Z), \\
& R^{(2)}(Z)=-\left(\frac{1}{6} \sqrt{2} Z-\frac{1}{12} Z^{2}\right) / f(Z),
\end{aligned}
$$

in which $\beta(Z)$ is another parallel transport parameter employed by Braginskii:

$$
\beta(Z)=\left(\frac{25}{16} \sqrt{2} Z+\frac{55}{48} Z^{2}\right) / f(Z)
$$

Physically $U_{a}^{(k)}$ is the solution appropriate to a fictitious plasma consisting of $N$ disparate-mass ion species, with densities $\bar{n}_{a}$ and changes $\bar{e}_{a}$ given by averages within the clumps. The appropriate average density is defined by 
$\bar{n}_{\mathbf{a}}=\rho_{\mathbf{a}}^{2} / \zeta_{\mathbf{a}}$, and the average charge by $\overline{\mathrm{e}}_{\mathbf{a}}=\zeta_{\mathbf{a}} / \rho_{\mathbf{a}}$.

Now we obtain the remaining part of the solution, $\bar{u}_{a i}^{(k)}-u_{a}^{(k)}$, which corresponds to the relative motion of charge states within the clump. Combining Eqs. (4.2) and (4.j), we see that this part of the solution satisfies

$$
\sum_{k^{\prime}} D^{k k^{\prime}}\left(z_{a}\right)\left[\bar{u}_{a i}^{\left(k^{\prime}\right)}-u_{a}^{\left(k^{\prime}\right)}\right]=\delta_{k 0} \frac{\zeta_{a}}{\lambda_{a a}}\left(\frac{1}{e_{a i}}-\frac{1}{\bar{e}_{a}}\right),
$$

where $\bar{e}_{a}$ is as defined above. Hence we have the simple result

$$
\bar{u}_{a i}^{(k)}=u_{a}^{(k)}+\left[D^{-1}\left(z_{a}\right)\right]^{k 0} \frac{\zeta_{a}}{\lambda_{a a}}\left(\frac{1}{e_{a i}}-\frac{1}{\bar{e}_{a}}\right) .
$$

Note that the only quantity on the right-hand side which refers to a particular member of the clump is the charge $e_{a i}$. In the three-polynomial approximation, the required matrix elements of $\mathrm{D}^{-1}$ work out to be

$$
\begin{aligned}
& {\left[D^{-1}(Z)\right]^{00}=\left(1.683+2.852 Z+0.5828 Z^{2}\right) / E(Z),} \\
& {\left[D^{-1}(Z)\right]^{10}=-\left(0.3965+1.121 Z+0.3545 Z^{2}\right) / E(Z),} \\
& {\left[D^{-1}(Z)\right]^{20}=\left(0.06143-0.03845 Z+0.06445 Z^{2}\right) / E(Z),}
\end{aligned}
$$

with

$$
E(Z)=1+2.612 z+1.343 z^{3}+0.1719 z^{3}
$$

Using a variational approach, Moore ${ }^{(13)}$ has calculated the parallel current for a system consisting of electrons and two ion species of arbitrary mass. Comparison with our results can be made in two cases: when the ion masses are equal, and when they are disparate. Very good agreement is found in each case. Moore does not determine the higher moments of the distribution function. 
We conclude this section by noting the formulas which give the Ware pinch and bootstrap current in terms of the Spitzer function, for a disparate-clump plasma. The Ware pinch coefficients $L_{13}^{\text {at }}$ and $L_{23}^{\text {af }}$ relate the radial fluxes of particles and heat to the induced toroidal electric field, according to

$$
\left(\begin{array}{cc}
\Gamma_{a 1} \\
\frac{1}{T_{a}} a_{a 1}
\end{array}\right)=\left(\begin{array}{c}
L_{13}^{a f} \\
L_{23}^{a 1}
\end{array}\right) \frac{\langle E \cdot B\rangle}{\left\langle B^{2}\right\rangle}+\sum_{b j}\left(\begin{array}{ll}
L_{11}^{a 1}, b j & L_{12}^{a 1}, b j \\
L_{21}^{a f}, b j & L_{22}^{a 1}, b j
\end{array}\right)\left(\begin{array}{c}
A_{1}^{b j} \\
A_{2}^{b}
\end{array}\right),
$$

with $A_{1}^{a 1}=\partial$ en $\mathrm{p}_{a 1} / \partial \psi$ and $A_{2}^{a 1}=\partial$ en $T_{a} / \partial \psi$. On the other hand, the bootstrap current, defined as the portion of the neoclassical current proportional to pressure and temperature gradients, has the expression

$$
\left\langle j_{n c}^{B}\right\rangle=\sum_{a 1} T_{a}\left(L_{31}^{a 1} A_{1}^{a i}+L_{32}^{a A_{2}^{a}}\right) .
$$

By the symmetry principle, we have $L_{m 3}^{a 1}=L_{3 m}^{a t}$ (for $m=1,2$ ) and so only one set of coefficients need be determined. Referring to the general formulas $(10)$ for the coefficients and performing a certain amount of algebra, we find that in a disparate-clump system:

$$
\begin{aligned}
L_{3 m}^{a i}= & (-1)^{m} f_{t} F \frac{\lambda_{a 1, a}}{e_{a 1}} \sum_{k} \bar{u}_{a i}^{(k)} j_{m-1, k}^{a i} \\
& -(-1)^{m_{f}} F_{t} \frac{\lambda_{a i}, a}{e_{a 1}} j_{m-1,0}^{a 1} \frac{\sum_{b j} \lambda_{b j, b} \sum_{k} \bar{u}_{b j}^{(k)} j_{0 k}^{b j}}{\sum_{b j} \lambda_{b j, b} j_{00}^{b j}},
\end{aligned}
$$

where $f_{t} \simeq 1.46 \varepsilon^{\frac{1}{2}}$ is the fraction of trapped particles, $\varepsilon$ is the inverse aspect ratio, $F(\psi)$ is the toroidal flux function, and 


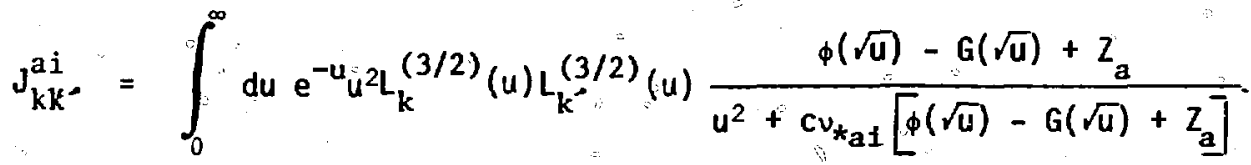

$$
\begin{aligned}
& \times\left\{1+c^{-} \varepsilon^{3 / 2} v_{\star_{a 1}} u^{-2}\left[\phi(\sqrt{u})+(8 u-3) G(\sqrt{u})+3 Z_{a}\right]\right\}^{-1} \text {. }
\end{aligned}
$$

with $\phi(x)$ the error function, $G(x)=-\frac{1}{2} \frac{d}{d x}[\phi(x) / x]$ the Chandrasekhar function, and

$$
c=\frac{4(1.46)}{\sqrt{\pi}}, \quad c^{-}=\frac{15 \pi^{3 / 2}}{32} .
$$

Finally, $v_{*_{a i}}$ is the collisionality index for species (ai):

$$
\nu_{\star_{\mathrm{a} i}}=\frac{\mathrm{R}_{0} q}{\varepsilon^{3 / 2} v_{\mathrm{Ta}}} \sum_{j} \nu_{\mathrm{ai}, \mathrm{aj}},
$$

with $R_{0}$ the major radius of the torus and $q$ the safety factor. The net toroidal flow $w$ does not appear in Eq. (4.29), since the general formula is independent of $w$.

\section{SOLUTION OF THE SPITZER PROBLEM - SECOND FORMULATION}

In this section we obtain the first two moments of the Spitzer function by an approach related more directly to that of Ref. $\{6\}$, in wich a three-term Laguerre polynomial expansion is employed throughout. Much of the notation in this section is taken from that reference. He first consider a single clump, and then generalize to a disparate-clump system. 


\section{A. Single-clump System}

We begin by defining

$$
n^{k \ell}=M_{i j}^{k \ell} / \zeta_{i} \zeta_{j}, \quad m^{k \ell}=M_{i}^{k \ell} / \zeta_{i}, \quad(i=1,2, \ldots, N),
$$

where $N$ is the number of species and $\zeta_{1}=n_{1} e_{1}^{2}$. In this new notation

$$
\Delta_{i j}=-N_{i j}^{22}\left(1-\delta_{1 j}\right) /\left(M_{i}^{22}+N_{i j}^{22}\right)=\left(-\underline{U} \underline{v}^{T}+D\right)_{i j},
$$

where

$$
U_{i} \equiv n^{22} \zeta_{i} /\left(M_{i}^{22}+N_{i 1}^{22}\right), \quad v_{i} \equiv \zeta_{i}, \quad D_{i} \equiv U_{i} V_{1} .
$$

From Eq. (5.2) one sees that

$$
(1-\Delta)^{-1}=\left(1+\tilde{D}^{-1} \underline{U} \underline{V}\right)^{-1} \tilde{D}^{-1},
$$

where $\tilde{D}=I-D$. Thus,

$$
\begin{aligned}
(1-\Delta)^{-1} & =\left[1-\frac{\tilde{D^{-1}} \underline{\underline{U}} \underline{V}^{T}}{1+\underline{v}^{T^{-1}}}\right] D^{-1} \\
& =\tilde{D}^{-1}-c_{1} \tilde{D}^{-1} \underline{U} \underline{V}^{T^{-}}-1
\end{aligned}
$$

Since $\left(\tilde{D}^{-1}\right)_{i i}=\left(M_{1}^{22}+N_{11}^{22}\right) / M_{i}^{22}$, it follows that

$$
\begin{aligned}
& D^{-1} \underline{U}=\left(n^{22} / \mathrm{m}^{22}\right) \underline{e}, \quad\left(e_{i}=1 ; i=1,2, \ldots, N\right) \\
& (1-\Delta)^{-1}=\left(I-c_{2} \underline{e} \underline{V}^{T}\right) \tilde{D}^{-1} .
\end{aligned}
$$

The form of the constants $c_{1}$ and $c_{2}$ (and of other such constants which appear, undefined, below) is not important here. 
It is easy to show that

$$
\begin{aligned}
& \alpha=\left(m^{20} / m^{22}\right) \tilde{D}+\left(n^{20} / n^{22}\right) \underline{\underline{V}} \underline{\underline{T}}^{\mathrm{T}}, \\
& \beta=\left(\mathrm{m}^{21} / \mathrm{m}^{22}\right) \tilde{D}+\left(\mathrm{n}^{21} / \mathrm{n}^{22}\right) \underline{\underline{U} \underline{v}^{\mathrm{T}} .}
\end{aligned}
$$

From Eqs. (5.7) and (5.8) it follows that

$$
\begin{aligned}
& \tilde{\alpha} \equiv(1-\Delta)^{-1_{\alpha}}=\left(m^{20} / \mathrm{m}^{22}\right) I+c_{3} \underline{e} \underline{V}^{\mathbf{T}}, \\
& \tilde{\beta}=\left(\mathrm{m}^{21 /} / \mathrm{m}^{22}\right) I+c_{4} \underline{e} \underline{v}^{\mathbf{T}} .
\end{aligned}
$$

Now

$$
\begin{aligned}
\ell^{22} & =m I\left[M^{11}-M^{12 \tilde{\beta}}+N^{11}-N^{12} \tilde{\beta}\right] \\
& =m I\left[M^{11}+N^{11}-\left(m^{21} / m^{22}\right)\left(M^{12}+N^{12}\right)+c_{4}\left(M^{12}+N^{12}\right) \leq V^{T}\right],(5.10) \\
\ell^{22} & =m I\left[\tilde{M}+c_{5} \underline{V} \underline{V}^{T}\right], \quad \tilde{M} \equiv M^{11}-\left(m^{21} / m^{22}\right) M^{12}
\end{aligned}
$$

It should be noted that $\tilde{M}$ is diagonal. From Eq. (5.11):

$$
\left(\ell^{22}\right)^{-1}=m^{-1} I\left[\tilde{M}^{-1}+c_{6} \tilde{M}^{-1} \underline{v} \underline{v}^{T} \tilde{M}^{-1}\right]=m^{-1} I\left[\tilde{M}^{-1}+c_{7} \underline{e} \underline{e}^{T}\right] .
$$

It is easy to show that

$$
\begin{aligned}
& \ell^{12}=M I\left[c_{8} \tilde{M}+c_{9} \unrhd V^{T}\right]=\ell^{21} \\
& c_{8} \equiv\left(-m^{01}+\frac{m^{21}}{m^{22}} m^{02}\right) /\left(m^{11}-\frac{m^{21}}{m^{22}} m^{12}\right) .
\end{aligned}
$$

Further

$$
\left(\ell^{22}\right)^{-1} \ell^{21}=c_{8} I+c_{10} \underline{\underline{V^{T}}} \underline{\underline{T}}^{\mathrm{T}} \text {. }
$$




$$
\ell^{12}\left(\ell^{22}\right)^{-1} \ell^{21}=m I\left[c_{8}^{2} \tilde{M}+c_{11} \underline{\underline{Y}} \underline{V}^{T}\right] .
$$

Also,

$$
\ell^{11}=m I\left[c_{12} \tilde{M}+c_{13} \underline{\underline{V}} \underline{\underline{T}}^{\mathrm{T}}\right] \text {, }
$$

where

$$
c_{12}=\left(m^{00}-\frac{m^{20}}{m^{22}} m^{02}\right) /\left(m^{11}-\frac{m^{21}}{m^{22}} m_{12}\right) .
$$

Combining Eqs. (5.15) and (5.16):

$$
H \equiv \ell^{11}-\ell^{12}\left(\ell^{22}\right)^{-1} \ell^{21}=m I\left[\left(c_{12}-c_{8}^{2}\right) \tilde{M}+c_{13} \underline{v} v^{\bar{T}}\right] \text {. }
$$

Here $c_{8}$ and $c_{12}$ have been defined explicitly, directly below Ens. (5.13) and (5.16). From Eq. (5.11):

$$
\tilde{M}_{1}=\tilde{c} S_{1}, \quad \tilde{c} \equiv m^{11}-\frac{m^{21}}{m^{22}} m^{12} .
$$

To compute $c_{13}$ explicitly we note that

$$
\mathbf{H} \underline{\mathbf{e}}=0 \text {. }
$$

From Ens. (5.17) through (5.19) it follows that

$$
\begin{aligned}
& \left(c_{12}-c_{8}^{2}\right) \tilde{c}+c_{13} \xi=0, c_{13}=-\left(c_{12}-c_{8}^{2}\right) \tilde{c} / S, \\
& \zeta \equiv \sum_{1} \zeta_{1}, \\
& H=\operatorname{mI}\left(c_{12}-c_{8}^{2}\right)\left(\tilde{M}-\frac{\tilde{c}}{\zeta} \underline{v} \underline{v}^{T}\right) .
\end{aligned}
$$

Parallel drift velocities in the classical Spitzes problem are determined by the equation

22 


$$
H \underline{u}^{\prime}=\underline{R}^{\prime \prime}, \quad R_{1}^{\prime}=n_{1} e_{1} E^{\prime} ;(i=1,2, \ldots, N) .
$$

Suppose that, in Eq. (5.22), we set $u_{N}^{\prime}=0$. "Then the other components of $u$ " will satisfy Eq. (5.23):

$$
H_{(t)} \stackrel{u}{\prime}(t)^{\prime}=\underline{R}_{(t)}^{\prime} .
$$

Here $H_{(t)}$ is an $(N-1) \times(N-1)$ matrix such that

$$
H_{(t) i, j}=H_{1, j}, \quad(i=1,2, \ldots . N-1 ; j=1,2, \ldots, N-1),
$$

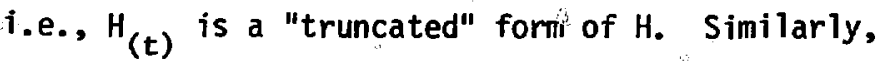

$$
u_{(t) i}^{\prime}=u_{i}^{\prime}, R_{(t) i}=R_{1} ;(i=1,2 \ldots, N-1) .
$$

It tan easily be shown that

$$
H_{(t)}^{-1}=m^{-1} I_{(t)}\left(c_{12}-c_{B}^{2}\right)^{-1}\left[\tilde{M}_{(t)}^{-1}+\frac{\left.e_{(t)}\right)^{T}(t)}{\tilde{c} \zeta_{N}}\right]
$$

so that

$$
u_{i}^{\prime}=E^{\prime} n^{-1}\left[e_{i}^{-1}-e_{N}^{-1}\right] /\left[\tilde{c}\left(c_{12}-c_{8}^{2}\right)\right] \text {. }
$$

Equation $(5.27)$ can also but in the form

$$
u_{i}^{\prime}=E^{\prime} K \frac{\zeta}{m \sum_{j k} n_{j} v_{j k}}\left(\frac{1}{e_{1}}-\frac{1}{e_{N}}\right)
$$


Evaluating the constant in Eq. (5.28), one finds that $K=1.6828$.

Given $\underline{u}^{\prime}, q^{\prime}$ can be determined from the relation

$$
0=\ell^{21} \underline{u}^{\prime \prime}+\ell^{22} \underline{q}^{\prime \prime}, \quad \tilde{q}_{i}^{\prime} \equiv q_{i}^{\prime \prime} / \nabla_{1} p_{i} .
$$

The calculation of $q^{\prime}$ does not, however, differ essentially from the calculation of $\underline{u}^{\prime \prime}$ and will not be carried out explicitly here. Both $\underline{u}^{\prime \prime}$ and q" wi11 be computed explicitly, in the next section, by a slightly different method.

We proceed now to the multiple clump case.

B. Properties of $\Delta, \alpha$, and $\beta$ for Disparate-Mass Species

We show first that $\Delta_{\mathbf{a} \sigma} \simeq \Delta_{\sigma a} \simeq 0$. Here a designates the lighter of two species, and $\sigma$ the heavier. We have

$$
\Delta_{a \sigma}=-\frac{x_{a \sigma} N_{a \sigma}^{22}}{M_{a}^{22}+N_{a a}^{22}} \sim x_{a \sigma}^{-4} \sim 0 .
$$

$$
\Delta_{\sigma a} \sim x_{\sigma a}^{5} \sim 0
$$

Since $\Delta_{\sigma \sigma}=0$, it follows that

$$
\hat{\alpha}_{\mathrm{a} \sigma} \simeq \alpha_{\mathbf{a \sigma}}, \quad \hat{\alpha}_{\sigma \alpha} \simeq \alpha_{\sigma \mathbf{a}}, \quad \beta_{\mathbf{a \sigma}} \simeq \beta_{\mathbf{a \sigma}}, \quad \hat{\beta}_{\sigma \mathbf{a}} \simeq \beta_{\sigma \mathbf{a}} .
$$

Next we show that $\beta_{a \sigma} \simeq f_{\sigma a} \simeq 0$. From the definiticn

$$
\beta_{a \sigma}=\frac{x_{a \sigma} N^{21}}{M_{a}^{22}+N_{a a}^{22}},
$$

and the observation 


$$
N_{a \sigma}^{21} \sim N_{\sigma a}^{12} \sim \frac{n_{\sigma} x_{\sigma a}^{2}}{\tau_{\sigma a}\left(1+x_{\sigma a}^{2}\right)^{7 / 2}} \sim \frac{n_{\sigma} x_{\sigma a}^{2}}{\tau_{\sigma a}}
$$

we indeed see that

$$
\beta_{a \sigma} \sim \frac{n_{\sigma} \tau_{a a}}{\tau_{\sigma a} n_{a}} x_{\sigma a} \sim\left(m_{a} / m_{\sigma}\right)^{\frac{1}{2}} x_{\sigma a} \sim x_{a \sigma}^{-2} \sim 0 .
$$

We also note that

$$
N_{\sigma a}^{21} \sim \frac{n_{a} x_{a \sigma}^{2}}{\tau_{a \sigma}\left(1+x_{a \sigma}^{2}\right)^{7 / 2}} \sim \frac{n_{a}}{\tau_{a \sigma}} x_{a \sigma}^{-5},
$$

and therefore

$$
B_{\sigma a} \sim \frac{n_{a} \tau \sigma \sigma}{\tau_{a \sigma} n_{\sigma}} x_{a \sigma}^{-6} \sim x_{a \sigma}^{-5} \sim 0
$$

In view of Eqs. $(5.30),(5.31),(5.35)$, and $(5.37)$, it will be convenient to refer to $\triangle$ and $\beta$ as "block diagonal". Next we show that $\alpha$ is block uppertriangular, since

$$
\alpha_{a \sigma}=\frac{x_{a \sigma} N_{a \sigma}^{20}}{M_{a}^{22}+N_{a a}^{22}} \sim \frac{x_{a \sigma} N_{\sigma a}^{02}}{M_{a}^{22}+N_{a a}^{22}} .
$$

But

$$
\begin{aligned}
& M_{a \sigma}^{02}=-\frac{m_{\sigma}}{m_{a}} x_{\sigma a} N_{\sigma a}^{02} \sim-x_{a \sigma} N_{\sigma a}^{02}, \\
& x_{a \sigma \sigma a} \sim-\frac{n_{a}}{\tau_{a \sigma}}\left(1+\frac{m_{a}}{m_{\sigma}}\right) \frac{x_{a \sigma}^{7}}{\left(1+x_{a \sigma}^{2}\right) 7 / 2} \sim-\frac{n_{a}}{\tau_{a \sigma}},
\end{aligned}
$$


so that

$$
\alpha_{\mathbf{a \sigma}} \simeq 1
$$

Similarly, we see that

$$
x_{\sigma a} N_{a \sigma}^{20} \sim-\frac{n_{\sigma} m_{\sigma}}{\tau_{\sigma a} m_{a}} x_{\sigma a}^{7},
$$

and so

$$
\alpha_{\sigma \mathbf{a}} \approx 0
$$

It can be shown, incidentally, that $\alpha_{\sigma \sigma} \simeq \alpha_{\sigma a} \simeq 0$ if $\sigma$ is the heaviest species in the plasma and a the next heaviest. In summary, we have at this point $\hat{\alpha}=\alpha, \hat{\beta}=\beta, \alpha_{\sigma a} \simeq 0, \alpha_{a \sigma} \simeq 1, \beta_{a \sigma} \simeq \beta_{\sigma a} \simeq 0$.

C. Computation of $\ell_{11}, \ell_{12}, \ell_{22}$, and $H$ We note, first, that the form of the matrix elements $\ell_{i j}$ is unaltered when a disparate mass species $\sigma$ is added to the plasma. of course the values of the matrix elements will change, since sums over the species now include species $\sigma$. More precisely, no new terms enter into the sums over $k$ in Eqs. (20) of Ref. 6. In Eq. (20c) such new terms would have to take the form $m_{a} x_{a \sigma^{N}}{ }^{12} \sigma_{\sigma b}$. Since $B$ is block-diagonal such terms vanish. Similarly one shows that no new terms occur in Eq. (20b). In Eq. (20a) new terms take the form $x_{a \sigma} N_{a \sigma}^{02} \alpha_{\sigma b}$. But if $m_{\sigma} \gg m_{b}$, then $\alpha_{\sigma b} \simeq 0$, and if $m_{\sigma} \ll m_{b}$ then $x_{a \sigma a \sigma} \cong 0$.

From now on the letters $\underline{a}, \underline{b}$, and $\sigma$ will designate clumps, and the letters 1, $j$, and $k$ will designate individual species. The matrix $H$, defined by

$$
H=\ell_{11}-\ell_{12} \ell_{22}^{-1} \ell_{21},
$$


has elements $H_{i \mathfrak{j}}^{a b}$ connecting species $\underline{\mathfrak{i}}$ in clump $\underline{a}$ with species $\underline{j}$ in clump $\underline{b}$. From what has been noted, above, one can show that the form of $H^{\text {aa }}$ is unaltered when disparate clumps $\sigma$ are added to the plasma. Thus we can show that it has the form

$$
H^{\mathrm{aa}}=m_{a}\left[C_{5}^{a}+\left(C_{3}^{a}\right)^{2}\right]\left[\tilde{\mu}^{a a}+\frac{c^{a}}{\zeta_{a}} \underline{v}^{a} \underline{v}^{a T}\right] \text {, }
$$

where

$$
v_{i}^{a}=n_{a i} e_{a i}^{2}
$$

and the C's are certain constants for a clump. In particular, we have

$$
\begin{aligned}
& c_{3}^{a}=\left(-m_{a}^{01}+\frac{m_{a}^{02} m_{a}^{21}}{m_{a}^{22}}\right) /\left(m_{a}^{11}-\frac{m_{a}^{12} m_{a}^{21}}{m_{a}^{22}}\right) \\
& m_{a}^{01}=\frac{1}{n_{a i} e_{a i}^{2}} M_{a i}^{01}, \quad i \in C_{a}, \\
& m_{a}^{02}=\frac{1}{n_{a i} e_{a i}^{2}} M_{a i}^{21}, \quad \text { etc. }
\end{aligned}
$$

The notation $i \in C_{a}$ means " $\underline{i}$ in clump $\underline{a}^{\prime}$. In the one-clump case the constant $C^{a}$, like $C^{a}$ can be determined from the condition

$$
\sum_{j \in C_{a}} H_{i j}^{a a}=0
$$

Now this is no longer possible. We do know, however, from explicit computations that $C^{a}, 1 i k e C_{5}^{a}$ and $C_{3}^{a}$, is a function only of the various clump masses, 
clump temperatures, and the quantities $\zeta_{b} / \zeta_{a}$ for $a l l \underline{b}$ [with $\zeta_{a}$ defined as in Eq. (4.4b)].

D. Computation of the Force on a Species: Parallel Velocities

Suppose that there are only two clumps, a and $\sigma$, in the plasma, with $m_{\sigma} \gg m_{a} \cdot$ Then

$$
\sum_{j \in C_{a}} H_{i j}^{a a}+\sum_{k \in C_{\sigma}} H_{i k}^{a v}=0 .
$$

From Eqs. (5.45) and (5.48) one can show that $\sum_{\mathbf{k} \in \mathrm{C}_{\sigma}} H_{\mathbf{i k}}^{\text {ao }}$ is proportional to $n_{a i} e_{a i}^{2}$. On physical grounds we know that it is also proportional to $n_{\sigma k} e_{\sigma k}^{2}$. - Hence

$$
H_{i k}^{a}=c^{a \sigma} n_{a i} e_{a i}^{2} n_{\sigma k} e_{\sigma k}^{2}
$$

Aga in from Eqs. (5.45) and (5.48) one can show that $c^{\text {ad }}$ can depend only on the clump masses, the clump temperatures, and the ratio $Z_{a}=\zeta_{\sigma} / \zeta_{a}$. But we know $H_{i k}^{a \sigma}$ when there is only one species per clump. In that case

$$
H^{a \sigma}=-m_{a} n_{a} v_{a \sigma}{ }^{\alpha}\left(z_{a}\right) \text {. }
$$

Therefore we conclude

$$
H_{i k}^{a \sigma}=-m_{a} n_{a i} \nu_{a i, \sigma k} \alpha\left(z_{a}\right) \text {. }
$$

The force $R_{i k}^{a \sigma}$ is a linear function of the drift velocities of all species such that $m_{k}>m_{1}$; it does not depend (directly) on the drift velocities of

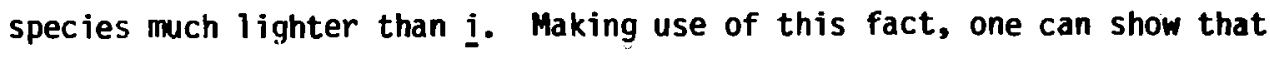
Eq. (5.51) is valid in general, no matter how many disparate clumps are present in the plasma. In this case $Z_{a}$ is defined as in Eq. (3.17). It follows that the net force $R^{a b}$ between clumps $\underline{a}$ and $\underline{b}$ is given by 


$$
R^{a b}=-m_{a} \bar{n}_{a} \nu_{a b} \alpha\left(z_{a}\right) \bar{u}_{a}
$$

for $b>a$. Here we have introduced the notation

$$
\begin{aligned}
\tilde{n}_{a} & =\frac{\rho_{a}^{2}}{\zeta_{a}}, \\
\bar{n}_{a} \nu_{a b} & =\sum_{i \in C_{a}} \sum_{j \in C_{b}} \bar{n}_{a i} \nu_{a i, b j}, \\
\bar{u}_{a} & =\frac{1}{\zeta_{a}} \sum_{i \in C_{a}} \bar{n}_{a i} e_{a i}^{2} u_{l i},
\end{aligned}
$$

with $P_{a}$ as in Eq. (4.6c).

Given Eq. (5.52) one find's that the clump-averaged velocities are given by

$$
R^{a}=-\rho_{a} E_{l}=-z_{a} m_{a} \bar{n}_{a} \nu_{a b} \alpha\left(z_{a}\right)^{\bar{u}}{ }_{a}-\sum_{b<a} R^{a b} .
$$

Thus the average velocities of the various clumps are determined as if each clump consisted of a single species with density given by Eq. (5.53), velocity given by Eq. (5.55), and charge given by $\bar{e}_{a}=\zeta_{a} / P_{a}$.

To compute the individual drift velocities we proceed as follows. First we note that

$$
R_{i}^{a}=\sum_{j \in C_{a}} H_{i j}^{a a} u_{a j}+\sum_{b \neq a} \sum_{k \in C_{b}} H_{i k}^{a b} u_{b k} .
$$

From Eq. (5.22) we know that the second term on the right-hand side is proportional to $n_{a 1} e_{a 1}^{2}$, so that we may write 


$$
R_{i}^{a}=\sum_{j \in C_{a}} H_{i j}^{a a} u_{a j}+\gamma_{a} n_{a i} e_{a i}^{2},
$$

where $\gamma_{a}$ is some constant whose value will not be needed. Therefore we have

$$
u_{a i}=\sum_{j \in C_{a}}\left(H^{a a}\right)_{i j}^{-1}\left(R_{i}^{a}-\gamma_{a} n_{a i} e_{a i}^{2}\right) \text {. }
$$

But by referring to a plasma consisting of a single clump, one can show that

$$
\left(H^{a a}\right)_{i j}^{-1}=\frac{\left(\tilde{M}^{a}\right)_{i j}^{-1}+\delta_{a}}{m_{a}\left[C_{5}^{a}+\left(C_{3}^{a}\right)^{2}\right]},
$$

where

$$
\tilde{M}_{i j}^{a}=\tilde{C}^{a} n_{a i}^{2} e_{a i} \delta_{i j}, \quad i \in C_{a} \text {, }
$$

and $\delta_{a}$ is a constant whose value is not needed.

Pltting Eq. (5.60) into Eq. (5.59) we find that the velocity has the form

$$
u_{a i}=\frac{E_{y}}{e_{a i} m_{a}\left[C_{5}^{a}+\left(C_{3}^{a}\right)^{2}\right]}+\epsilon_{a i} .
$$

Finally the constant $\varepsilon_{a i}$ can be determined from the condition

$$
\frac{1}{\zeta_{a}} \sum_{i \in c_{a}} n_{a i} e_{a i}^{2} u_{a i}=\bar{u}_{a} \text {. }
$$

When this is done we conclude that

$$
u_{a i}-\bar{u}_{a}=K_{a} \frac{\zeta_{a} E_{1}}{\lambda_{a a}}\left(\frac{1}{e_{a i}}-\frac{\rho_{a}}{\zeta_{a}}\right) .
$$

with $\lambda_{\text {aa }}$ as in Eq. (4.6b) and $K_{a}$ a dimensionless constant. Comparing Eq. (5.64) with the earlier result, Eq. (4.24), we haye $K_{a}=\left[D^{-1}\left(z_{a}\right)\right]^{00}$. 


\section{E. Random Heat Fluxes}

From the $u_{a 1}$ 's we compute the parallel heat flow $q_{a i}$ via

$$
\left.\frac{q_{a 1}}{p_{a i}}=-\sum_{b} \sum_{j \in c_{b}}\left(e_{22}^{-1} \ell_{21}\right)\right)_{i j}^{a b} u_{b j}, \quad i \varepsilon c_{a} .
$$

Because $\ell_{21}$ is upper triangular and $u_{b j} \ll u_{a f}$ for $m_{b} \gg m_{a}$, we have

$$
\frac{q_{a i}}{p_{a i}}=-\sum_{j \in C_{a}}\left(\ell^{-\frac{1}{2} \ell^{21}}\right)_{i j}^{a a} u_{a j} .
$$

But by referring to a one-clump calculation, one can show that

$$
\left.\left(\ell_{22}^{-1} \ell_{21}\right)\right)_{i j}^{a a}=C_{3}^{a}\left(\delta_{i j}-\xi_{a} n_{a j} e_{a j}^{2}\right),
$$

where $\xi_{a}$ is a constant whose value will not be needed. Thus, from Eqs. (5.66) and (5.67), we see that the heat flux has the form

$$
\frac{q_{a 1}}{p_{a 1}}=C_{3}^{a} u_{a 1}+n_{a} .
$$

Arguing as before, one can show that the quantity

$$
\bar{h}_{a}=\frac{1}{\zeta_{a}} \sum_{i \in c_{a}} n_{a i} e_{a i}^{2} \frac{q_{a i}}{p_{a i}}
$$

is the same as in the case in which each clump is replaced by a single "equivalent" species, with density, velocity, and charge as given earlier in this section. Thus we conclude that

$$
\frac{q_{a 1}}{p_{a 1}}-\bar{h}_{a}=c_{3}^{a}\left(u_{a 1}-\bar{u}_{a}\right) \text {, }
$$

and, referring to the more comprehensive result, Eq. (4.24), we have 


$$
C_{3}^{a}=\frac{\left[D^{-1}\left(z_{a}\right)\right]^{10}}{\left[D^{-1}\left(z_{a}\right)\right]^{00}}
$$

VI. DIFFUSION COEFFICIENTS IN THE PFIRSCH-SCHLÜTER REGIME

Now we return to the kinetic equation with inhomogeneity appropriate to the diffusion problem, Eq. (2.6b). We shall be content with the case $k_{\max }=2$. Eliminating $u^{(2)}$, we arrange Eq. $(2.6 b)$ as a system for the first two moments alone:

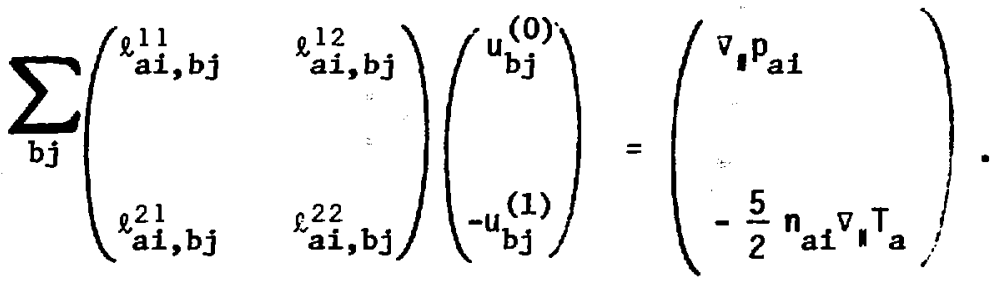

where

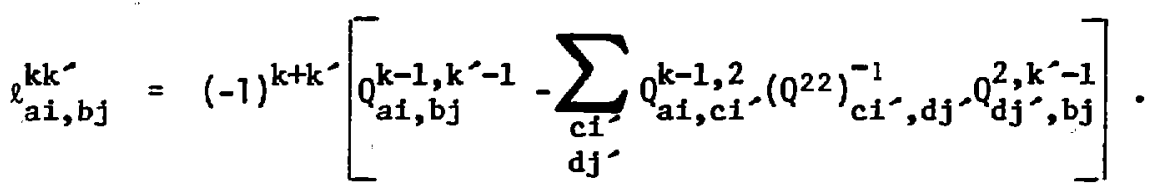

These quantities, with the indicated sign convention, are called the classical friction coefficients. By conservation of momentum, we have

$$
\sum_{a i}^{\ell_{a f, b j}^{11}}=\sum_{a i}^{l_{a f, b j}^{12}}=0 .
$$

In the Pfirsch-Schlüter regime, the friction coefficients completely determine the radial fluxes. (6) The form of the results depends on the choice of temper, Gures which have equilibrated along the magnetic field line. We shall be concerned with the case in which all the ions have equilibrated to a common 
temperature $T$ and in which the electrons have a different temperature $T_{e} \cdot B y$ a straightforward extension of the procedure developed in the single-temperature case, ${ }^{(6)}$ we obtain the following expressions for the electron flux and the individual ion fluxes:

$$
\begin{aligned}
& r_{e}^{P S}=\frac{1}{e} G_{1}\left[l_{e e}^{11} \frac{p_{e}^{\prime}}{n_{e} e}+l_{e e}^{12} \frac{T_{e}^{e}}{e}-\sum_{a 1} l_{e, a 1}^{11} \frac{p_{a 1}^{-}}{n_{a 1} e_{a 1}}\right], \\
& \Gamma_{a 1}^{P S}=\frac{1}{e_{a 1}} G_{1}\left[-l_{a 1, e}^{11} \frac{p_{e}^{-}}{n_{e} e}-l_{a 1, e}^{12} \frac{T e}{e}+\frac{1}{L} S L_{a 1} T^{-}\right. \\
& \left.+\sum_{b j}\left(L_{a i, b j}+\frac{1}{L} L_{a 1} L_{b j}\right) \frac{p_{b j}^{-}}{n_{b j} e_{b j}}\right] \text {, }
\end{aligned}
$$

and for the electron heat flux $q_{e}$ and the (total) ion heat flux $q$ :

$$
\begin{aligned}
& q_{e}^{P S}=G_{1} \frac{T e}{e}\left[l_{e e}^{21} \frac{p_{e}^{-}}{n_{e}^{e}}+l_{e e}^{22} \frac{T_{e}^{e}}{e}-\sum_{a i} l_{e, a 1}^{21} \frac{p_{a 1}^{-}}{n_{a 1} e_{a 1}}\right], \\
& q^{P S}=G_{1} \frac{S T}{L}\left[\sum_{a i} L_{a i} \frac{p_{a 1}^{\prime}}{n_{a 1} e_{a 1}}+S T-\right.
\end{aligned}
$$

where the prime denotes $\partial / \partial \psi$ and from now on the indices (ai) refer only to the ions. The L's denote the following matrix products:

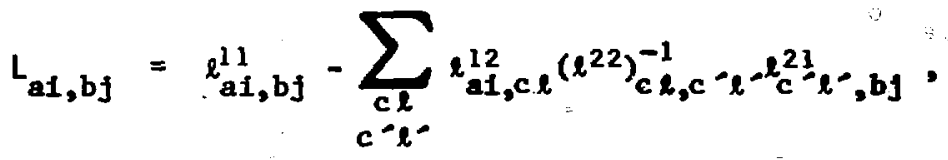




$$
\begin{aligned}
L_{a 1} & =\sum_{\substack{b j \\
c l}} l_{a 1, b j}^{12}\left(\ell^{22}\right)_{b j, c \ell^{n} c l}^{-1}, \\
L & =\sum_{a i} n_{a i}\left(l^{22}\right)_{a i, b j}^{-1} n_{b j} .
\end{aligned}
$$

We have also defined

$$
\begin{aligned}
S & =\sum_{a 1} \frac{n_{a i}}{e_{a 1}}, \\
G_{1} & =F^{2}\left(\left\langle\frac{1}{B^{2}}\right\rangle-\frac{1}{\left\langle B^{2}\right\rangle}\right),
\end{aligned}
$$

the latter being the characteristic Pfirsch-Schlüter geometrical factor for a magnetic field of the assumed form, $\vec{B}=F(\psi) \vec{\nabla} \phi+\vec{\nabla} \psi$. In arriving at Eqs. (6.4)(6.7), we have made use of the simplifying features that $\ell_{e, a i}^{12}=\ell_{a i, e}^{21}=\ell_{e, a i}^{22}$ $=\ell_{\text {ai,e }}^{22}=0$. It will be noted that the particle fluxes satisfy the ambipolarity condition $\mathrm{er}=\sum_{\mathrm{ai}} \mathrm{e}_{\mathrm{ai}} \mathrm{r}_{\mathrm{al}}$

Equations $(6.8)-(6.70)$ specify the particular combinations of classical friction coefficients which are needed for the radial fluxes. Those involving electrons are easy to work out:

$$
\begin{aligned}
\ell_{e e}^{l 1} & =-\lambda e^{Z} c_{1}^{e}, \\
\ell_{e, a i}^{11} & =\ell_{a i, e}^{11}=\lambda_{e, a i} C_{1}^{e}, \\
\ell_{e e}^{12} & =l_{e e}^{21}=\lambda_{e e} Z_{e} C^{e}, \\
l_{a i, e}^{12} & =l_{e, a i}^{21}=-\lambda_{e, a i} C_{2}^{e}, \\
l_{e e}^{22} & =-\lambda_{e e} C_{3}^{e},
\end{aligned}
$$


where $c_{i}^{e}=c_{i}^{*}\left(z_{e}\right)$, the functions noted in Ref. 6:

$$
\begin{aligned}
& c_{1}^{*}(z)=\frac{1}{z}\left[c^{00}-\frac{\left(c^{02}\right)^{2}}{c^{22}}\right]=1-\frac{\left(\frac{15}{8}\right)^{2} z}{\frac{45}{16} \sqrt{2}+\frac{433}{64} z}, \\
& c_{2}^{*}(z)=\frac{1}{z}\left[c^{01}-\frac{c^{02} c^{2} 2}{c^{22}}\right]=\frac{3}{2}-\frac{15}{8} \frac{\frac{3}{4} \sqrt{2}+\frac{69}{16} z}{\frac{45}{16} \sqrt{2}+\frac{433}{64} z}, \\
& c_{3}^{*}(z)=c^{11}-\frac{\left(c^{12}\right)^{2}}{c^{22}}=\sqrt{2}+\frac{13}{4} z-\frac{\left(\frac{3}{4} \sqrt{2}+\frac{69}{16} z\right)^{2}}{\frac{45}{16} \sqrt{2}+\frac{433}{64} z},
\end{aligned}
$$

with $C^{k k^{*}}(Z)$ as in Eq. (4.6a).

The friction coefficients for the ion species are somewhat more complicated. We find

$$
\begin{aligned}
& e_{a 1, b j}^{11}=-\delta_{a b} \delta_{1 j}\left[\lambda_{a, a 1} D_{1}^{a}+\sum_{c<a} \lambda_{c, a 1}+\lambda_{e, a 1}\right] \\
& -\delta_{a b a j, a j} \lambda_{a j}\left(Z_{a} C_{1}^{a}-D_{1}^{a}\right) \\
& +\theta_{a b}{ }_{a i, b j} C_{1}^{a}+\theta_{b a} \lambda_{b j, a i} C_{1}^{b} \\
& +\zeta_{a i} \zeta_{b j}\left[\sum_{\substack{c<a \\
c<b}} \frac{\lambda_{c c}}{\zeta_{c}^{2} c_{c}}\left(1-c_{1}^{c}\right)+\frac{\lambda_{e e}}{\zeta_{e}^{2} Z_{e}}\left(1-c_{1}^{e}\right)\right] .
\end{aligned}
$$




$$
\begin{aligned}
l_{a 1, b j}^{12} & =\delta_{a b} \delta_{i j} \lambda_{a, a 1} D_{2}^{a}+\delta_{a b} \lambda_{a 1, a j}\left(Z_{a} C_{2}^{a}-D_{2}^{a}\right)-\theta_{b a} \lambda_{b j, a i} C_{2}^{b} \\
& =e_{b j, a 1}^{21}, \\
l_{a f, b j}^{22} & =-\delta_{a b} \delta_{i j} \lambda_{a, a 1} D_{3}^{a}-\delta_{a b} \lambda_{a f, b j}\left(C_{3}^{a}-D_{3}^{a}\right) .
\end{aligned}
$$

where $D_{i}^{a}=D_{i}\left(Z_{a}\right),(i=1,2,3)$, with

$$
\begin{aligned}
& D_{1}(Z)=D^{00}-\frac{\left(D^{02}\right)^{2}}{D^{22}}, \\
& D_{2}(Z)=D^{01}-\frac{D^{02} D^{21}}{D^{22}}, \\
& D_{3}(Z)=D^{11}-\frac{\left(D^{12}\right)^{2}}{D^{22}},
\end{aligned}
$$

in terms of the matrix $D^{k k^{\prime}}$, defined in Eq. (3.19). It can be checked that the following sums vanish, in accordance with the conservation of momentum:

$$
\begin{aligned}
\ell_{e, b j}^{\underline{l} 1}+ & +\sum_{a i}^{l_{a i, b j}^{11}}=0 \\
\sum_{a f} l_{a 1, b j}^{12} & =0 .
\end{aligned}
$$

In the case in which each clump consists of a single species, the friction coefficients coincide with those given in Ref. 6 .

The particular combinations of ion friction coefficients which appear in the radial fluxes work out to be 


$$
\begin{aligned}
& L_{a i, b j}=-\delta_{a b} \delta_{1 j}\left[\lambda_{a, a 1} D^{a}+\sum_{c<a} \lambda_{c, a i}+\lambda_{e, a i}\right] \\
& -\delta_{a b} \lambda_{a 1, a j}\left(Z_{a} \alpha_{a}-D_{4}^{a}\right) \\
& +\theta_{a b}{ }_{a i, b j}{ }^{\alpha} a+\theta_{b a} \lambda_{b j, a i}{ }^{\alpha} b \\
& +\zeta_{a 1} \zeta_{b j}\left[\sum_{\substack{c<a \\
c<b}} \frac{\lambda_{c c}}{\zeta_{c}^{2} Z c}\left(1-\alpha_{c}\right)+\frac{\lambda_{e e}}{\zeta_{e e}^{2} Z}\left(1-c_{1}^{e}\right)\right] \text {, } \\
& L_{a 1}=-\frac{\zeta_{a i}}{\zeta_{a}} n_{a} Z_{a} \frac{c_{2}^{a}}{c_{3}^{a}}-\left(n_{a}-\frac{\zeta_{a i}}{\zeta_{a}} n_{a}\right) D_{5}^{a} \\
& +\zeta_{a 1} \sum_{b<a} \frac{n_{b} c_{2}^{b}}{\zeta_{b} c_{3}^{b}} \\
& L=-\sum_{a} \frac{n_{a}^{2}}{\lambda_{a a}}\left(\frac{1}{c_{3}^{a}}+\frac{\phi_{a}^{(1)}}{D_{3}^{a}}\right),
\end{aligned}
$$

where $n_{a}$ is the density of a clump:

$$
n_{a}=\sum_{i} n_{a 1},
$$

and $\phi_{a}^{(1)}$ is the first of two averages involving the charge distribution within a clump which we wi11 need:

$$
\phi_{a}^{(1)}=\frac{1}{2 n_{a}^{2}} \sum_{i j}{ }_{a i}^{n}\left(\frac{e j}{e_{a j}}-\frac{e j}{e_{a j}}\right)^{2} \text {. }
$$

The quantities $\mathrm{D}_{4}^{\mathrm{a}}$ and $\mathrm{D}_{5}^{\mathrm{a}}$ are defined by 


$$
D_{4}^{a}=D_{1}^{a}-\frac{\left(D_{2}^{a}\right)^{2}}{D_{3}^{a}}, \quad D_{5}^{a}=\frac{D_{2}^{a}}{D_{3}^{a}},
$$

and, in terms of the functions in Eqs. (4.25) and (4.26), are given explicitly by

$$
\begin{aligned}
& D_{4}^{a}=\frac{1}{\left[D^{-1}\left(z_{a}\right)\right]^{00}}, \\
& D_{5}^{a}=-\frac{\left[D^{-1}\left(Z_{a}\right)\right]^{10}}{\left[D^{-1}\left(Z_{a}\right)\right]^{00}} .
\end{aligned}
$$

We will also include the classical fluxes in the discussion, since they are comparable in size to the PS fluxes (except for large values of the safety factor). They can be written in simple analytic form even for arbitrary clump masses. We have $(14,15)$

$$
\begin{aligned}
\Gamma_{a i}^{c l}= & -G_{2} \frac{m_{e} n_{e}^{\nu} e_{, a i}}{e_{a i}}\left(\frac{p_{e}^{\prime}}{n_{e} e}+\frac{p_{a i}^{-}}{n_{a i} e_{a i}}-\frac{3 T_{e}^{\prime}}{2 e}\right) \\
& -G_{2} \sum_{b j} \frac{m_{a} n_{a i} \nu_{a i}, b j}{e_{a i}\left(1+\beta_{a b}^{2}\right)^{1 / 2}}\left(\frac{p_{a i}^{\prime}}{n_{a i} e_{a i}}-\frac{p_{b j}^{-}}{n_{b j} e_{b j}}\right) \\
& +\frac{3}{2} G_{2} T^{-} \sum_{b j}\left[\frac{m_{a} n_{a i} \nu_{a i, b j}}{e_{a i}^{2}\left(1+\beta_{a b}^{2}\right)^{3 / 2}}-\frac{m_{b} n_{b j} \nu_{b j, a i}}{e_{a i} e_{b j}\left(1+\beta_{b a}^{2}\right)^{3 / 2}}\right],
\end{aligned}
$$




$$
\begin{aligned}
& \frac{1}{T} q^{c l}=\frac{3}{2} G_{2} \sum_{a i j} \frac{m_{a j} n_{a i} \nu_{a i, b j}}{e_{a i}\left(1+\beta_{a b}^{2}\right)^{3 / 2}}\left(\frac{p_{a i}^{\prime}}{n_{a i} e_{a i}}-\frac{p_{b j}^{\prime}}{n_{b j} e_{b j}}\right) \\
& -\frac{1}{4} G_{2} T \cdot \sum_{a i} \frac{m_{b} n_{b j} \nu_{b j, a i}}{e_{a j}\left(1+\beta_{a b}^{2}\right)^{5 / 2}}\left[\frac{1}{e_{a i}}\left(30 \beta_{a b}^{5}+16 \beta_{a b}^{3}+13 \beta_{a b}\right)-\frac{27 \beta_{a b}^{3}}{e_{b j}}\right],(6.2) \\
& \frac{1}{T_{e}} q_{e}^{c l}=G_{2} m_{e} n_{e}\left[\frac{3}{2} \sum_{a i} \nu_{e, a i}\left(\frac{p_{e}^{-}}{n_{e} e}+\frac{p_{a i}^{-}}{n_{a i} e_{a i}}\right)+\left(\frac{13}{4}+\frac{\sqrt{2}}{Z_{e}}\right) \frac{Z_{e} \nu_{e e}}{e}\right]
\end{aligned}
$$

where $\beta_{a b}^{2}=m_{a} / m_{a}$ and $G_{2}$ is the characteristic geometrical factor for classical transport in a torus:

$$
G_{2}=\left\langle\frac{|\nabla \psi|^{2}}{B^{2}}\right\rangle
$$

Taking the disparate-mass limit of the classical fluxes and combining these expressions with the Pfirsch-Schliuter fluxes found earlier, we find that the total fluxes can be written in the form

$$
\begin{aligned}
& \Gamma_{a i}=\frac{n_{a i} e_{a i}}{n_{e} e}\left[\frac{1}{n_{e}} I_{e}^{(1)} p_{e}^{-}+I_{e}^{(2)} T e j+\frac{1}{n_{e}} I_{a} p_{a i}^{-}\right.
\end{aligned}
$$

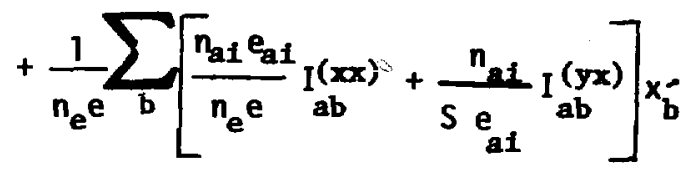

$$
\begin{aligned}
& +\frac{1}{S} \sum_{b}\left[\frac{n_{a i} e_{a i}}{n_{e} e} I_{a b}^{(x y)}+\frac{n_{a i}}{S e_{a i}} I_{a b}^{(y y)}\right] y_{b}^{-}
\end{aligned}
$$




$$
\begin{aligned}
& +\left[\frac{n_{a 1}}{n_{e}} I_{a}^{(0)}+\frac{n_{a 1} e_{a 1}}{n_{e} e} I_{a}^{(1)}+\frac{1}{S} \frac{n_{a 1}}{e_{a 1}} I_{a}^{(2)}\right] T^{-} \\
& T_{e}=\left(\frac{Z_{e}}{n_{e}}+\frac{T}{p_{e}}\right) I_{e}^{(1)} p_{e}^{\prime}+\left(Z_{e}^{I} I_{e}^{(2)}-\frac{T}{T_{e}} I_{e}^{(1)}\right) T_{e}^{\prime}+I_{e}^{(1)} T^{-}, \\
& \frac{1}{T} q=\sum\left[\frac{1}{n_{e}} I_{a}^{(0)} p_{a}^{-}+\frac{1}{n_{e} e^{(1)}} x_{a}^{-}+\frac{1}{S} I_{a}^{(2)} y_{a}^{-}\right]+I^{-}, \\
& \frac{1}{T_{e}} q_{e}=\left(\frac{Z_{e}}{n_{e}}+\frac{T}{p_{e}}\right) I_{e}^{(2)} p_{e}^{\prime}+\left(I_{e}^{(3)}-\frac{T}{T_{e}} I_{e}^{(2)}\right) T_{e}^{\prime}+I_{e}^{(2)} T^{\prime},
\end{aligned}
$$

in which two auxiliary clump variables have been introduced:

$$
\begin{aligned}
& x_{a}=\sum_{i} e_{a 1} p_{a 1}, \\
& y_{a}=\sum_{i} \frac{p_{a 1}}{e_{a 1}} .
\end{aligned}
$$

After a great deal of algebra, the coefficients appearing in the expressions for the fluxes are found to be

$$
\begin{aligned}
& I_{e}^{(1)}=-G_{1} \frac{C_{1}^{e} \lambda_{e e}}{e^{2}}-G_{2} \frac{\lambda_{e e}}{e^{2}}, \\
& I_{e}^{(2)}=G_{1} \frac{C_{2}^{e} \lambda_{e e}}{e^{2}}+G_{2} \frac{3 \lambda}{2 e_{e}} \\
& 2 e^{2} \\
& I_{e}^{(3)}=-G_{1} \frac{C_{3 \lambda}^{e} e_{e}}{e^{2}}-G_{2} \frac{\lambda_{e e}}{e^{2}}\left(\sqrt{2}+\frac{13}{4} Z_{e}\right),
\end{aligned}
$$




$$
\begin{aligned}
& I_{a}=-\frac{n_{e}}{\zeta_{a}}\left[\lambda_{a a} G_{1} D_{4}^{a}+G_{2} \lambda_{a a}\left(Z_{a}+\frac{1}{\sqrt{2}}\right)+\left(G_{1}+G_{2}\right)\left(\sum_{c<a} \lambda_{c a} \lambda_{e a}\right)\right],(6.38) \\
& I_{a b}^{(x x)}=G_{1}\left(n_{e} e\right)^{2}\left[-\delta_{a b}\left(Z_{a} a_{a}-D_{4}^{a}\right) \xi_{a}+\theta_{a b} \alpha_{a^{5} a^{\prime}}+\theta_{b a^{\alpha} b_{b}}\right. \\
& \left.+\sum_{c} \theta_{c a}{ }^{\theta} c b \frac{\xi_{c}}{Z_{c}}\left(1-\alpha_{c}\right)+\frac{\xi_{e}}{Z_{e}}\left(1-c^{e}\right)+\frac{1}{L} A_{a} A_{b}\right] \\
& +G_{2}\left(n_{e} e\right) 2\left(\frac{1}{\sqrt{2}} \delta_{a b} \xi_{a}+\theta_{a b} \xi_{a}+\theta_{b a} \xi_{b}\right), \\
& I_{a b}^{(y x)}=-G_{1} \frac{n_{e}^{e S}}{L} D_{5}^{a_{A}} A_{b}, \\
& =\mathrm{I}_{\mathrm{ba}}^{(\mathrm{xy})} \text {, } \\
& I_{a b}^{(y y)}=G_{1} \frac{S^{2}}{L} D_{5}^{a} D_{5}^{b}, \\
& I_{a}^{(0)}=G_{2} \frac{3 n_{e}}{2} \frac{\lambda_{a a}}{\zeta_{a}}\left(Z_{a}+\frac{1}{2 \sqrt{2}}\right), \\
& I_{a}^{(1)}=n_{e} e\left[G_{1} \frac{S}{L} A_{a}-\frac{G_{2}}{T}\left(\frac{3}{4 \sqrt{2}} \xi_{a} x_{a}+\frac{3}{2} \sum_{b<a} \xi_{b} x_{b}\right)\right] \text {, } \\
& I_{a}^{(2)}=-G_{1} \frac{S^{2}}{L} D_{5}^{a}, \\
& I=G_{1} \frac{S^{2}}{L}-G_{2}\left[\frac{27}{16 \sqrt{2}} \sum_{a} \xi_{a} x_{a}^{2}{ }_{a}^{(2)}+\sqrt{2} \sum_{a} \frac{\lambda_{a a_{a}}{ }^{n}}{\zeta_{a}}\right] \text {, }
\end{aligned}
$$

where 


$$
\begin{aligned}
& A_{a}=-\frac{c_{2}^{a} n_{a} Z_{a}}{C_{3}^{a} \zeta_{a}}+D_{5}^{a} \frac{n_{a}}{\zeta_{a}}+\sum_{b<a} \frac{C_{2}^{b} n_{b}}{C_{3}^{b} r_{b}}, \\
& \xi_{a}=\frac{4(2 \pi)^{1 / 2} \ell n \Lambda}{3\left(4 \pi \varepsilon_{0}\right)^{2}} \frac{m_{a}^{1 / 2}}{T^{3 / 2}}=\left(\frac{m_{a}}{m_{e}}\right)^{1 / 2}\left(\frac{T_{e}}{T}\right)^{3 / 2} \xi_{e},
\end{aligned}
$$

and $\phi_{a}^{(2)}$, like $\phi_{a}^{(1)}$, is a measure of the charge distribution within a clump:

$$
\phi_{a}^{(2)}=\left(\sum_{i j} n_{a i} e_{a i}\right)^{-2} \frac{1}{2} \sum_{i j} n_{a i} n_{a j}\left(e_{a i}-e_{a j}\right)^{2} .
$$

The coefficients $I$ in Eqs. (6.35)-(6.45) have been normalized so that all have the same dimensions. It will be noticed that some of them appear more than once, in consequence of the reciprocity relations for the original fluxes. The electron flux, Eq. (6.31), is consistent with the ambipolarity condition.

Althougn the fluxes, as given in Eqs. $(6.30)-(6.33)$ seem rather complicated at first sight, it will be noticed that only three combinations of "internally averaged" variables (those containing a sum over charge states) occur within the gradients. In addition, the pressure gradient term in $r_{a i}$ has the property that the internal index appears explicitly only within the gradient. These features suggest an efficient method for obtaining numerical solutions of the one-dimensional transport equations. First, though, we recall the form of the transport equations themselves:

$$
\frac{\partial n_{a i}}{\partial t}+\frac{1}{r} \frac{\partial}{\partial r}\left(r \tilde{r}_{a i}\right)=s_{a i}
$$




$$
\begin{aligned}
& \frac{\partial}{\partial t}\left(\frac{3}{2} n T\right)+\frac{1}{r} \frac{\partial}{\partial r} r\left(\tilde{q}+\frac{5}{2} T \sum_{a i} \tilde{\Gamma}_{a i}\right)=-P_{1}-P_{2}, \\
& \frac{\partial}{\partial t}\left(\frac{3}{2} n_{e} T\right)+\frac{1}{r} \frac{\partial}{\partial r} r\left(\tilde{q}_{e}+\frac{5}{2} T_{e} \tilde{r}_{e}\right)=\frac{1}{n} E^{2}+P_{1}+P_{2},
\end{aligned}
$$

where for simplicity we work with a magnetic field having concentric, circular flux surfaces:

$$
\vec{B}=\frac{\hat{\phi} B_{\phi_{0}}(r)+B_{\theta 0}(r)}{1+\left(r / R_{0}\right) \cos \theta} \text {, }
$$

with $\phi$ the azimuthal angle, $\theta$ the poloidal angle, $r$ the distance from the magmetic axis, and $R_{0}$ the major radius of the torus. The flux $\tilde{\Gamma}_{\text {ai }}$ is obtained from $\Gamma_{\text {al }}$ via the substitutions

$$
\begin{aligned}
& G_{1}+\tilde{G}_{1} \equiv \frac{B_{\phi 0}^{2}}{B_{\theta 0}^{2}\left(B_{\theta 0}^{2}+B_{\phi 0}^{2}\right)}\left[1+\frac{3}{2} \varepsilon^{2}-\left(1-\varepsilon^{2}\right)^{1 / 2}\right], \\
& G_{2}+\tilde{G}_{2} \equiv \frac{1}{B_{\phi 0}^{2}+B_{\theta 0}^{2}}\left(1+\frac{3}{2} \varepsilon^{2}\right), \\
& \frac{\partial}{\partial \psi}+\frac{\partial}{\partial r},
\end{aligned}
$$

with $\varepsilon=r / R_{0}$. The quantity $S_{a i}$ is the coronal source term for ion a in the isth ionization state:

$$
S_{a 1}=n_{e}\left[\alpha_{a, 1-1} n_{a, 1-1}+\beta_{a 1} n_{a, 1+1}-\left(\alpha_{a 1}+\beta_{a, 1-1}\right) n_{a 1}\right] \text {, }
$$

43 
with $\alpha_{a 1}$ and $\beta_{a 1}$ the ionization and recombination coefficients. The energy sources are the Ohmic heating term (with $E$ the induced electric field), the temperature equilibration term,

$$
P_{1}=-3\left(T_{e}-T\right) \frac{\lambda_{e e}}{\zeta_{e}} \sum_{a i} \frac{\zeta_{a 1}}{m_{a}},
$$

and a Pfirsch-Schlüter source discussed by Hirshman and Sigmar:

$$
\begin{aligned}
P_{2}= & \left(\tilde{G}_{1}+\tilde{G}_{2}\right) \frac{\lambda_{e e}}{\zeta_{e}} \sum_{a 1} \zeta_{a i}\left[\frac{1}{n_{a i} e_{a i}} \frac{\partial p_{a i}}{\partial r}-\frac{1}{n_{e} e Z} \frac{\partial\left(n_{e} T\right)}{\partial r}\right]^{2} \\
& -\frac{1}{n_{e} Z} \frac{\partial\left(n_{e} T\right)}{\partial r} \tilde{\Gamma}_{e} .
\end{aligned}
$$

The parallel resistivity in the Ohmic heating term is given by the Spitzer formula for an impure plasma:

$$
\eta=\frac{\alpha_{e}^{Z} e^{m} e_{e e}^{\nu}}{\zeta_{e}}
$$

To discuss the method of solution mentioned in the previous paragraph, we need the diffusion equations for $n_{a}, x_{a}$, and $y_{a}$. Multiplying Eq. (6.49) successively by unity, $e_{a i}$, and $e_{a i}^{-1}$ and summing over $i$, we have 


$$
\begin{aligned}
\frac{\partial n_{a}}{\partial t}+\frac{1}{r} \frac{\partial}{\partial r} & r\left\{\frac{x_{a}}{n_{e} e T}\left[\frac{j}{n_{e}} \tilde{I}_{e}^{(1)} \frac{\partial p_{e}}{\partial r}+\tilde{I}_{e}^{(2)} \frac{\partial T_{e}}{\partial r}\right]+\frac{1}{n_{e}} \tilde{I}_{a} \frac{\partial\left(n_{a} T\right)}{\partial r}\right. \\
& +\frac{1}{n_{e} e T} \sum_{b}\left[\frac{x_{a}}{n_{e} e} \tilde{I}_{a b}^{(x x)}+\frac{y_{a}}{S} \tilde{I}_{a b}^{(y x)}\right] \frac{\partial x_{b}}{\partial r} \\
& +\frac{1}{S T} \sum_{b}\left[\frac{x_{a}}{n_{e} e} \tilde{I}_{a b}^{(x y)}+\frac{y_{a}}{S} \tilde{I}_{a b}^{(y y)}\right] \frac{\partial y_{b}}{\partial r} \\
+ & {\left.\left[\tilde{I}_{a}^{(0)}+\frac{x_{a}}{n_{e} e T} \tilde{I}_{a}^{(1)}+\frac{y_{a}}{S T} \tilde{I}_{a}^{(2)}\right] \frac{\partial T}{\partial r}\right)=0, }
\end{aligned}
$$

$$
\begin{aligned}
\frac{\partial}{\partial t}\left(\frac{x_{a}}{T}\right)+\frac{1}{r} & \frac{\partial}{\partial r} r\left\{\frac{\zeta_{a}}{n_{e} e}\left[\frac{1}{n_{e}} \tilde{I}^{(1)} \frac{p_{e}}{r}+\tilde{I}_{e}^{(2)} \frac{T_{e}}{r}\right]\right. \\
& +\frac{1}{n_{e} e} \sum_{b}\left[\frac{n_{e}^{e}}{n_{e}} \delta_{a b} \tilde{I}_{a}+\frac{\zeta_{a}}{n_{e} e} \tilde{I}_{a b}^{(x x)}+\frac{n_{a}}{S} \tilde{I}_{a b}^{(y z)}\right] \frac{\partial x_{b}}{\partial r} \\
& +\frac{1}{S} \sum_{b}\left[\frac{\zeta_{a}}{n_{e} e} \tilde{I}_{a b}^{(x y)}+\frac{n_{a}}{S} \tilde{I}_{a b}^{(y y)}\right] \frac{\partial y_{b}}{\partial r} \\
& \left.+\left[\frac{x_{a}}{n T} \tilde{I}_{a}^{(0)}+\frac{\zeta_{a}}{n_{e} e} \tilde{I}_{a}^{(1)}+\frac{n_{a}}{S} \tilde{I}_{a}^{(2)}\right] \frac{\partial T}{\partial r}\right\}=\sum_{1} e_{a i} S_{a 1},
\end{aligned}
$$




$$
\begin{aligned}
& \frac{\partial}{\partial t}\left(\frac{y_{a}}{T}\right)+\frac{1}{r} \frac{\partial}{\partial r} r\left\{\frac{n_{a}}{n_{e}}\left(\frac{1}{n_{e}} \ddot{I}_{e}^{(1)} \frac{\partial p_{e}}{\partial r}+\tilde{I}_{e}^{(2)} \frac{\partial T}{\partial r}\right)\right. \\
& +\frac{1}{n_{e} e} \sum_{b}\left[\frac{n_{a}}{n_{e} e} \tilde{I}_{a b}^{(x x)}+\frac{\bar{s}}{s} \tilde{I}_{a b}^{(y x)}\right] \frac{\partial x_{b}}{\partial r} \\
& +\frac{1}{s} \sum_{b}\left[\frac{s}{n_{e}} \delta_{a b} \tilde{I}_{a}+\frac{n_{a}}{n_{e} e} \tilde{I}_{a b}^{(x y)}+\frac{\bar{s}}{s} \tilde{I}_{a b}^{(y y)}\right] \frac{\partial y_{b}}{\partial r} \\
& \left.+\left[\frac{S}{n} \tilde{I}_{a}^{(0)}+\frac{n_{a}}{n_{e} e} \tilde{I}_{a}^{(1)}+\frac{\bar{s}}{S} \tilde{I}_{a}^{(2)}\right] \frac{\partial T}{\partial r}\right\}=\sum_{i} \frac{S}{e_{a i}},
\end{aligned}
$$

where

$$
\bar{s}=\sum_{a f} \frac{n_{a i}}{e_{a i}^{2}}
$$

and the tilda over the I's means that the geometrical factors are taken as $\tilde{G}_{1}$ and $\tilde{G}_{2}$. We will also need the energy diffusion equations in the explicit form:

$$
\begin{aligned}
\frac{\partial}{\partial t}\left(\frac{3}{2} n T\right)+ & \frac{I}{r} \frac{\partial}{\partial r} r\left\{\frac{5}{2} T\left[\frac{1}{n_{e}} \tilde{I}_{e}^{(1)} \frac{\partial r_{e}}{\partial r}+\tilde{I}_{e}^{(2)} \frac{\partial T}{\partial r}\right]\right. \\
& +\frac{T}{n_{e}} \sum_{b}\left[\tilde{I}_{b}^{(0)}+\frac{5}{2} \tilde{I}_{b}\right] \frac{\partial\left(n_{b} T\right)}{\partial r} \\
& +\frac{T}{n_{e} e} \sum_{b}\left[\tilde{I}_{b}^{(1)}+\frac{5}{2} \sum_{a}\left(\frac{x_{a}}{n_{e} e^{T}} \tilde{i}_{a b}^{(x x)}+\frac{y_{a}}{S T} \tilde{I}_{a b}^{(y x)}\right)\right] \frac{\partial x_{b}}{\partial r}
\end{aligned}
$$




$$
\begin{aligned}
& +\frac{T}{S} \sum_{b}\left[\tilde{I}_{b}^{(2)}+\frac{5}{2} \sum_{a}\left(\frac{x_{a}}{n_{e} e T} \tilde{I}_{a b}^{(y x)}+\frac{y_{a}}{S T} \tilde{I}_{a b}^{(y y)}\right)\right] \frac{\partial y_{b}}{\partial r} \\
& \left.+T\left[\tilde{I}+\frac{5}{2} \sum_{a}\left(\frac{n_{a}}{n_{e}} \tilde{I}_{a}^{(0)}+\frac{x_{a}}{n_{e} e T} \tilde{I}_{a}^{(1)}+\frac{y_{a}}{S T} \tilde{I}_{a}^{(2)}\right)\right] \frac{\partial T}{\partial r}\right\}=-P_{1}-P_{2}, \\
\frac{\partial}{\partial t} \frac{3}{2}\left(n_{e}{ }^{T}\right) & +\frac{1}{r} \frac{\partial}{\partial r}\left\{\frac{1}{n_{e}}\left(Z_{e} \cdot T_{e}+T\right)\left(\frac{5}{2} \tilde{I}_{e}^{(1)}+\tilde{I}_{e}^{(2)}\right) \frac{\partial p}{\partial r}\right. \\
& +\left[-\frac{5}{2} T \tilde{I}_{e}^{(1)}+\left(\frac{5}{2} z_{e}^{T}-T_{e}\right) \tilde{I}_{e}^{(2)}+T_{e} \tilde{I}_{e}^{(3)}\right] \frac{\partial T}{\partial r} \\
& \left.+\left(\frac{5}{2} \tilde{I}_{e}^{(1)}+\tilde{I}_{e}^{(2)}\right) T_{e} \frac{\partial T}{\partial r}\right\}=\frac{1}{n} E^{2}+P_{1}+P_{2} \cdot
\end{aligned}
$$

Equations (6.49) and (6.58)-(6.62) are the basis of the following discussion. As was shown by Okamoto and Amano, (17) the numerical solution of diffusion equations with coronal source terms can be reduced to the solution of two simpler problems. In one problem one solves the diffusion equations, without coronal terms, over a time step by any appropriate method (say, a CrankNicholson scheme); in the other problem one solves the coronal rate equations, by an eigenvalue method, within the time step. It is to the first problem that the special features of the disparate-clump fluxes are relevant. To solve the plasma diffusion equations, without coronal terms, we proceed as follows. The variables $n_{a 1}, T$, and $T_{e}$ are denoted at the beginning of the $j$-th time step by $n_{a 1}^{(j)}, T^{(j)}$, and $T_{e}^{(j)}$; these quantities are presumed to be known. By extrapolating obserived trends, we guess the values $n^{(j+1)}, T^{(j+1)}$, and 
$T_{e}^{(j+1)}$ at the end of the time step. Next we form the averages

$$
\bar{n}_{\mathrm{ai}}^{(j)}=\frac{1}{2}\left[n_{\mathrm{ai}}^{(j+1)}+n_{\mathrm{ai}}^{(j)}\right] .
$$

Using these averages we compute mesh-centered values for the diffusion coefficients in Eqs. (6.49) and (6.58)-(6.62). Given these diffusion coefficients, we solve Eqs. (6.58)-(6.62) simultaneously (except that the energy source $P_{2}$ is taken from the previous time step, since it contains gradients of all the unknowns). If a particular atomic species, say species $\underline{a}$, contains fewer than three charge states, then the equations giving the evolution of $n_{a}, x_{a}$, and $y_{a}$ are not linearly independent, and only one or two of these equations need be included. Thus the total number of equations which must be solved simultaneously is:

$$
N=\sum_{a} N_{a}+2,
$$

where $N_{a}$ is the number of charge states or $N_{a}=3$, whichever is smaller.

Once these equations are solved, we insert the resulting values of $n_{a}$, $x_{a}, y_{a}, T$, and $T_{e}$ (along with $n_{a 1}^{(j+1)}$ ), into the right-hand side of Eq. (6.49) and solve for the increment in $n_{a 1}$. Now the entire process is iterated to the derived level of convergence.

It will be noted that this method of solution is a Crank-Nicholson method, specialized to take advantage of the particular form of the fluxes. Regardless of the number of species present in the plasma, only $\mathrm{N}$ diffusion equations are solved simultaneously; the remaining equation for $n_{a i}$ is then totally decoupled. On the other hand, without the disparate-clump approximation, the number of diffusion equations which must be solved simultaneously is given by the total number of individual charge states plus two. Obviously this exceeds $N$ if any ion has more than three charge states. 


\section{REFERENCES}

1. TFR Group, Phys. Rev. Letters 36, 1306 (1976).

2. V. Arunasalam et a1., "Recent Results from the PLT Tokamak," PPPL-1436, Princeton Plasma Physics Laboratory (1978).

3. S. P. Hirshman, D. J. Sigmar and J. F. Clarke, Phys. Fluids 19, 656 (1976).

4. C. D. Boley, E. M. Gelbard and W. M. Stacey, Jr., Phys. Fluids 19, 2051 (1976).

5. S. P. Hirshman and D. J. Sigmar, Phys. Fluids 20, 418 (1977).

6. S. P. Hirshman, Phys. Fluids 20, 589 (1977).

7. C. D. Boley, "Summary of Neoclassical Transport Coefficients for a Multispecies, Mixed-Regime Tokamak Plasma," ANL/FPP/TM-101, Argonne National Laboratory (1977).

8. E. M. Gelbard and S. P. Hirshman, Fhys. Fluids 21, 145 (1978).

9. S. P. Hirshman, Phys. Fluids 21, 224 (1978).

10. S. P. Hirshman and A. H. Boozer, "Neoclassical Current in a TorcidallyConfined Multispecies Plasma," PPL-1409, Princeton Plasma Physics Laboratory (1977); S. P. Hirshman, Phys. Fluids (to be published).

11. Most of the results of this section can be inferred from Ref. 6 .

12. S. I. Braginskii, in Reviews of Plasma Physics, M. A. Leontovich, Ed. (Consultants Bureau, New York, 1965), Vo1. I, p. 205.

13. T. B. Moore, "Transport Properties of a Tokamak Plasma with Impurities," FRCR-122, Fusion Research Center, University of Texas at Austin (1976).

14. S. P. Hirshman, Sc.D. Thesis, Massachusetts Institute of Technology (1976). 
15. C. D. Boley, in "CTR Quarterly Progress Report, April-Jure 1976," ANL/CTR-76-4 (1976), p. 36.

16. S. P. Hirshman and D. J. Sigmar, Nucl. Fusion (to be published).

17. M. Okamoto and T. Amano, "A Method to Solve Impurity Diffusion Equation," Memo 6828, Japan Atomic Energy Research Institute (1976). 


\section{Internal:}

M. Abdou

P. Amundson

W. P. Barthold

M. Benson

P. B. Bertoncini

C. D. Boley

J. N. Brooks

Y. Chang

D. A. Ehst

K. Evans, Jr.

E. M. Gelhord
M. Y. Gohar

L. R. Greenwood

D. M. Gruen

J. Jung

H. Henryson

M. Kaminsky

R. Kustom

L. LeSage

v. Jaroni

$\mathrm{J}$. Norem

F. Nolfi

P. J. Persiani
A. B. Smith

W. J. Sturm

C. E. Till

A. Travelli

D. C. Wade

C. Hytry (20)

FP Program (2)

A. B. Krisciunas ANL Contract File

ANL Libraries (5)

TIS Files (6)

\section{External :}

DOE-TIC, for distribution per UC-20a and $-20 \mathrm{~g}$ (214)

Manager, Chicago Operations Office

Chief, Office of Patent Counsel, $\mathrm{CH}$

President, Argonne Universities Association

Applied Physics Division Review Committee:

P. W. Dickson, Jr., Westinghouse Electric Corp.

R. 'L. Hellens, Combustion Engineering, Inc.

W. B. Loewenstein, Electric Power Research Inst.

R. F. Redmond, Ohio State U.

R. liver, Stanford U.

D. B. Wehmeyer, Detroit Edison Co.

S. A. Werner, U. Missouri

E. C. Crume, Oak Ridge National Lab.

S. P. Hirshman, Oak Ridge National Lab.

J. T. Hogan, Oak Ridge National Lab.

F. L. Hinton, U. Texas, Austin

W. W. Pfeiffer, General Atomic

D. E. Post, Princeton Plasma Physics Lab.

J. N. Davidson, Georgia Inst. Techology

W. M. Stacey, Georgia Inst. Technology

T. Uckan, Oak Ridge National Lab.

C. E. Wagner, Science Applications, Inc.

R. E. Waltz, General Atomic 